\title{
The appraisal of chronic stress and the development of the metabolic syndrome: a systematic review of prospective cohort studies
}

\author{
N Bergmann', F Gyntelberg ${ }^{2}$ and J Faber ${ }^{1,3}$ \\ ${ }^{1}$ Endocrine Unit, Department of Medicine O, Herlev University Hospital, DK-2730 Herlev, Denmark \\ ${ }^{2}$ The National Research Centre for the Working Environment, Copenhagen, Denmark \\ ${ }^{3}$ Faculty of Health Sciences, Copenhagen University, Copenhagen, Denmark
}

Correspondence

should be addressed

to $\mathrm{N}$ Bergmann

Email

n.c.bergmann@hotmail.com

\begin{abstract}
Chronic psychosocial stress has been proposed as a risk factor for the development of the metabolic syndrome (MES). This review gives a systematic overview of prospective cohort studies investigating chronic psychosocial stress as a risk factor for incident MES and the individual elements of MES. Thirty-nine studies were included. An association between chronic psychosocial stress and the development of MES was generally supported. Regarding the four elements of MES: i) weight gain: the prospective studies supported etiological roles for relationship stress, perceived stress, and distress, while the studies on work-related stress (WS) showed conflicting results; ii) dyslipidemi: too few studies on psychosocial stress as a risk factor for dyslipidemia were available to draw a conclusion; however, a trend toward a positive association was present; iii) type 2 diabetes mellitus (DM2): prospective studies supported perceived stress and distress as risk factors for the development of DM2 among men, but not among women, while WS was generally not supported as a risk factor among neither men nor women; iv) hypertension: marital stress and perceived stress might have an influence on blood pressure (BP), while no association was found regarding distress. Evaluating WS the results were equivocal and indicated that different types of WS affected the BP differently between men and women. In conclusion, a longitudinal association between chronic psychosocial stress and the development of MES seems present. However, the number of studies with sufficient quality is limited and the design of the studies is substantially heterogeneous.
\end{abstract}

\author{
Key Words \\ - metabolic syndrome \\ - adiposity \\ - hypertension \\ - dyslipidemia \\ - type 2 diabetes mellitus \\ - psychological stress \\ - job stress
}

\section{Introduction}

The metabolic syndrome (MES) is a cluster of risk factors including male adiposity, dyslipidemia, reduced glucose tolerance and hypertension. MES is highly prevalent and increasing in most parts of the world (1). A meta-analysis has shown that MES increases the risk of myocardial

http://www.endocrineconnections.org DOI: 10.1530/EC-14-0031 Published by Bioscientifica Ltd (c) 2014 The authors infarction, cardiovascular disease, and cardiovascular disease mortality by twofold (1). Therapies based on lifestyle intervention, especially weight loss, are the main focus in treating MES. However, the etiology seems multifactorial, which calls for new areas of prevention

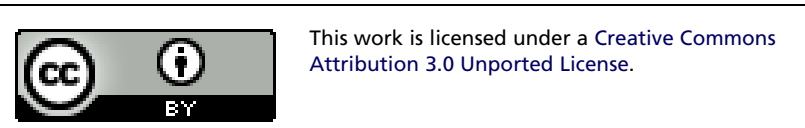


and intervention. One potential risk factor for the development of MES is chronic psychosocial stress, in the following referred to as stress.

In general it is accepted to divide the stress-concept into stressors (strain), stress (the bodily reaction to stressors), and distress (the emotional consequences as reactions to the stressors) (2). Stress occurs when the sum of stressors exceeds an individual threshold. The stress-response is then initiated with activation of the hypothalamic-pituitary-adrenal (HPA) axis and the sympathetic-adrenomedullar (SAM) axis (3). The activation of the two axes takes place in order to maintain the dynamic balance of the body, homeostasis, and is a necessary defence mechanism in situations of acute stress. However, if stress persists a state designated allostatic-load or chronic stress is reached, which among other things results in distress, reduced quality of life, or depression (4). There is no general consensus on how to measure chronic stress. Some have used physiological changes from the activation of the HPA- and SAM-axes as measures of stress: i.e. cortisol, noradrenalin, blood pressure (BP), or resting pulse (5). However, these parameters fluctuate, have great inter-individual variability, and reflect mostly acute stress-load (6). For these reasons, we will not consider the physiological stress-measures as markers of chronic psychosocial stress in this review.

Others have used the number of stressors as a measure of exposure. Example: the number of major life events or hours of work (7). However, the capacity for coping with stressors is dependent on the balance between e.g. the perception of the stressor, individual resources, and copingmechanisms, and thus the appraised stress caused by the exposure to stressors varies greatly between individuals. This makes the number of stressors by themselves of questionable value as measures of stress. Thus, in the following, we exclude studies measuring only stressors, and focus on studies measuring the appraisal of stress, including reactions to both single and multiple stressors.

The best measure for quantifying the effect of chronic stress is so far by questionnaires (Qs). A variety of Qs have been used for the evaluation of stress. Outside of the working field the most frequently used scale is the Perceived Stress Scale by Cohen. It measures the degree to which situations in one's life are appraised as stressful rated by ten questions on feelings and thoughts during the last month (8). In the occupational area, the most widely used Q's are the Job Demand Control model by Karasek et al. (9) and the Effort-Reward Imbalance model by Siegrist et al. $(10,11)$, which both are usable for evaluating work stress (WS). Another way of evaluating stress is by measuring distress which emerges when the allostatic load is reached for the individual subject and only when stress has been in progress for some time. Distress is mostly evaluated by the general health questionnaire (GHQ) by Goldberg \& Williams (12), measuring the inability to carry out normal functions and the appearance of distressing experiences.

The aim of the present review is to draw a synthesis from prospective cohort studies, in which chronic stress, measured by Qs, has been evaluated as potential risk factors for the development of MES as well as for the individual components of MES.

\section{Subjects and methods}

\section{Search strategy}

A systematic search in PubMed was done using the following keywords and their combinations: Metabolic syndrome, Abdominal fat, Body mass index, Weight gain, Weight change, Obesity, adiposity, Dyslipidemia, Cholesterol, Blood pressure, Hypertension, Hyperglycemia, Insulin resistance, Diabetes mellitus, Blood glucose, Psychological stress, Psychosocial stress, Work stress, Job stress, Strain, Prospective, Cohort, and Longitudinal.

Web of Science was used to search for references and citations from selected articles.

Articles were limited to human studies from February 1990 to October 2013 written in English. Bibliographies of relevant citations were screened for further articles of relevance.

\section{Criteria for inclusion}

In order to evaluate the causality between chronic stress and the elements of the MES, we included only prospective cohort studies. Studies were included if stress was evaluated by Qs, measuring the appraisal of stress in general (perceived stress) by Q's measuring, e.g. stress from relationships, by appraised WS or by standardized Qs on WS as e.g. effort-reward imbalance or job strain (job demands/control imbalance).

The outcome of the studies should be the diagnosis of MES defined according to general accepted classifications (i.e. from WHO, NCEP, and IDF; Box 1) or by each of the individual components of MES: adiposity, reduced glucose tolerance, dyslipidemia, or elevated blood pressure.

Studies were limited to adults ( $>18$ years of age). When more than two data-categories were reported, the extremes were compared, i.e. 'highest' vs 'lowest' category of perceived stress, thus excluding the intermediate groups.

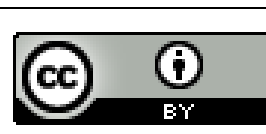

This work is licensed under a Creative Commons Attribution 3.0 Unported License. 


\begin{tabular}{|c|c|c|}
\hline $\begin{array}{l}\text { International Diabetes Foundation } \\
\text { (IDF) (7) }\end{array}$ & $\begin{array}{l}\text { the U.S. National Cholesterol } \\
\text { Education Program (NCEP) Adult } \\
\text { treatment Panel -III (ATP-III) (7) }\end{array}$ & $\begin{array}{l}\text { World Health Organization } \\
\text { (WHO) (7) }\end{array}$ \\
\hline $\begin{array}{l}\text { WC } \geq 94 \mathrm{~cm} \text { for Europid men } \\
\text { and } \geq 80 \mathrm{~cm} \text { for Europid } \\
\text { women }\end{array}$ & & $\begin{array}{l}\text { - fasting plasma glucose } \\
>6.1 \mathrm{mM} \text { or taking } \\
\text { medication for diabetes }\end{array}$ \\
\hline plus any two of the following: & $\geq 3$ of the following: & plus any two of the following: \\
\hline $\begin{array}{l}\text { Triglycerides: } \geq 1.7 \mathrm{mM} \text {, or } \\
\text { specific treatment for this }\end{array}$ & $\begin{array}{l}\text { - WC }>120 \mathrm{~cm} \text { in men and } \\
>88 \mathrm{~cm} \text { in women }\end{array}$ & 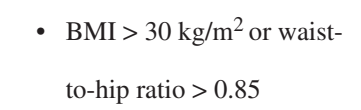 \\
\hline lipid abnormality & - Triglycerides $\geq 1.7 \mathrm{mM}$ & - Triglycerides $\geq 1.7 \mathrm{mM}$ \\
\hline $\begin{array}{l}\text { HDL cholesterol: }<1.03 \mathrm{mM} \\
\text { in men }<1.29 \mathrm{mM} \text { in women, } \\
\text { or specific treatment for this }\end{array}$ & $\begin{array}{l}\text { HDL cholesterol: }<1.0 \mathrm{mM} \\
\text { in men and }<1.3 \mathrm{mM} \text { in } \\
\text { women, }\end{array}$ & $\begin{array}{l}\text { - HDL cholesterol }<0.9 \mathrm{mM} \\
\text { in men, }<1.0 \mathrm{mM} \text { in } \\
\text { women }\end{array}$ \\
\hline $\begin{array}{l}\text { lipid abnormality } \\
\text { - } \quad \text { systolic } \mathrm{BP} \geq 130 \text { or diastolic }\end{array}$ & $\begin{array}{l}\text { systolic } \mathrm{BP} \geq 130 \text { and/or } \\
\text { diastolic } \mathrm{BP} \geq 85 \mathrm{~mm} \mathrm{Hg}\end{array}$ & $\begin{array}{r}\text { - Systolic } \mathrm{BP} \geq 140 \mathrm{and} / \mathrm{or} \\
\text { diastolic } \mathrm{BP} \geq 90 \mathrm{~mm} \mathrm{Hg}\end{array}$ \\
\hline $\begin{array}{l}\mathrm{BP} \geq 85 \mathrm{~mm} \mathrm{Hg} \text {, or treatment } \\
\text { of previously diagnosed } \\
\text { hypertension }\end{array}$ & $\begin{array}{l}\text { Fasting plasma glucose } \\
\geq 6.1 \mathrm{mM}\end{array}$ & \\
\hline $\begin{array}{l}\text { fasting plasma glucose } \geq 5.6 \\
\mathrm{mM} \text {, or previously diagnosed } \\
\text { type } 2 \mathrm{DM}\end{array}$ & & \\
\hline
\end{tabular}

\section{Box 1}

Clinical criteria for the metabolic syndrome.

When models with different numbers of adjustments were available, the most adjusted model was included excluding the intermediate analyses. When analyses were separated by gender, the individual gender results were included rather than the results based on mixed gender analyses.

\section{Criteria for exclusion}

Studies covering prenatal stress, childhood adverse events, depression, anxiety, hostility, fatigue, or personality types were excluded as were studies measuring the number of stressful life events. Studies measuring physiological stress (such as oxidative stress, cold or heat-shock responses) and studies evaluating stress by a physiological measure such as cortisol or noradrenalin were also excluded. With regard to WS, stress measured as physically challenging work, shift work, or hours of work was excluded to keep the focus on the psychosocial working conditions.

When later follow-up studies were published on the same cohort, any previous reports on the cohort addressing the same subject were excluded unless different exposure or outcome was measured.

The literature review conformed to Preferred Reporting Items for Systematic Reviews and Meta-analysis http://www.endocrineconnections.org DOI: 10.1530/EC-14-0031 (c) 2014 The authors Published by Bioscientifica Ltd
This work is licensed under a Creative Commons Attribution 3.0 Unported License. 
(PRISMA) statement standards (13). The heterogeneity of study designs and the limited numbers of studies available precluded a meta-analysis.

\section{Results}

Thirty-nine studies were eligible and included. The characteristics and findings of the studies are presented in Tables 1, 2, 3, 4 and 5.

\section{Association between stress and MES}

Seven prospective cohort studies that measured MES as the outcome variable were included: one study evaluated the exposure to global perceived stress (7), two studies evaluated marital stress $(14,15)$, one study evaluated psychological distress (16), while three studies evaluated the exposure to different measures of WS $(17,18,19)$.

The studies not specifically measuring WS were based on two different cohorts: two studies were based on data from The Healthy Women Study Cohort, while one study was based on a cohort of middle-aged white subjects from Finland.

Marital dissatisfaction $>$ On data from the Healthy Women Cohort, a study evaluated the 413 women who had answered seven questions on marital quality (14). The study found that after 11.5 years of follow-up, marital dissatisfied women had a three times higher risk of developing MES as defined by NCEP as compared with marital satisfied women (14). Another study based on 216 married couples, living in England, evaluated marital adjustment and found that husbands' report of poorer marital adjustment at baseline were associated with ten times greater risk of wives having MES as defined by NCEP after 4 years of follow-up (15). However, the wives' reports of poorer marital adjustment did not have significant influence on husbands' risk of developing MES (15).

Perceived stress $\downarrow$ From The Healthy Women Study Cohort, a tendency of a higher risk of developing MES as defined by NCEP and IDF was also found among subjects with perceived stress, evaluated in 523 women after 15 years of follow-up (7). When defining MES by the WHO-5 definitions, the association became statistically significant (7).

Distress - From the study based on the Finnish cohort, 466 middle-aged men and women demonstrated that a high score of psychological distress at baseline, evaluated by the General health $Q$, almost doubled the risk of developing MES as defined by NCEP after a mean of 6.4 years of follow-up (16). The difference remained significant after adjusting for example, socioeconomic status, lifestyle factors, and depression score (16).

Work stress - Three studies evaluated WS based on two different cohorts: Two studies were based on the Whitehall II cohort, consisting of working men and women from 20 civil services departments in London. One study was based on the CARDIA study cohort of citizens from Birmingham, Chicago, Minneapolis, and Oakland.

Based on the Whitehall II cohort, one study demonstrated a significantly dose-dependent association between self-perceived stress at work and the risk of developing MES as defined by NCEP (17). The analyses were not separated by gender. The other Whitehall II study investigated feeling of justice at work, and separating the analyses by gender, they found that a high feeling of justice was associated with a lower risk of developing MES during 18-year follow-up among men, but not among women (18). On the CARDIA study cohort, job strain was evaluated among 1408 men and 1558 women. The study showed that after adjusting for sociodemographic factors, health behaviors and depressive symptoms, women in high strain jobs (high demands/low control) had a significantly increased risk of MES compared with women in low strain jobs after 5 years of follow-up (19). The same trend was found among men; however, the association was only borderline significant (19).

Conclusion - The studies included support an association between perceived stress and distress and the development of MES. Marital stress was also found to be a risk factor, especially among women. However, only one study measuring stress outside of the working area separated the analyses by gender, and two out of four studies included only women. Concerning WS, perceived stress at work was supported as a risk factor for MES. A high feeling of justice at work was suggested as a protective factor among men, while job strain was found to be a risk factor for MES among women. These findings indicate that the sexes might respond differently to different kinds of stress exposures.

\section{Association between stress and the single elements of MES}

Stress and adiposity $\bullet$ Nineteen prospective cohort studies evaluating adiposity were included. One study evaluated the exposure to negative aspects of close

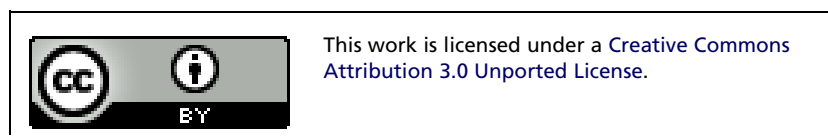




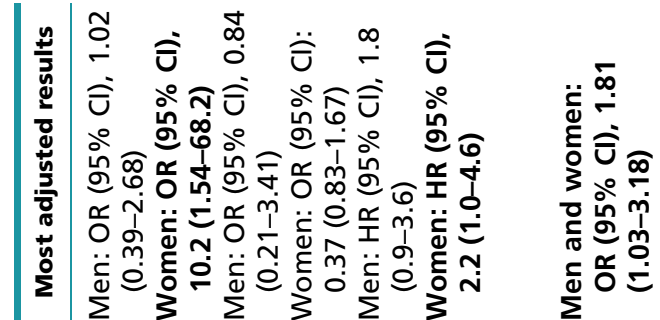

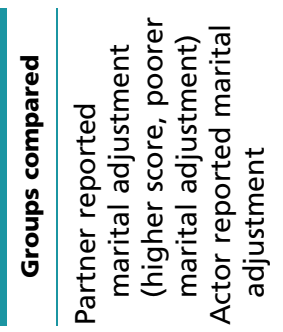

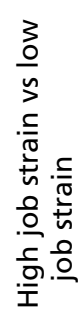

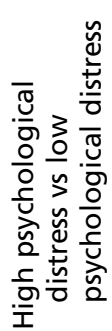

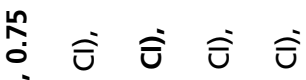

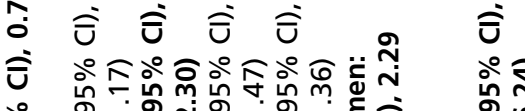

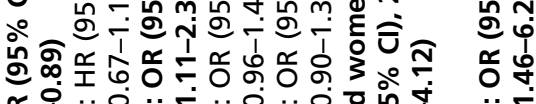

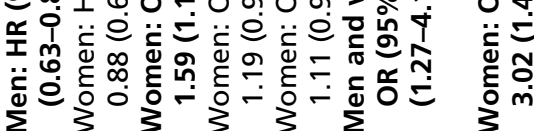

\begin{tabular}{|c|}
\hline 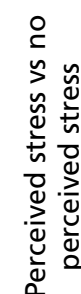 \\
\hline
\end{tabular}

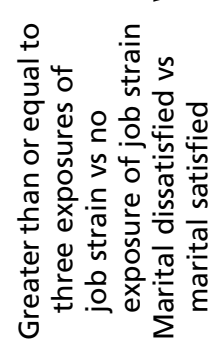

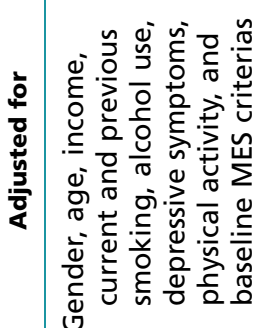

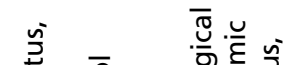

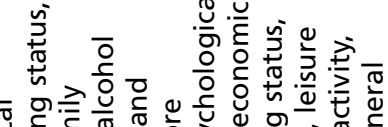

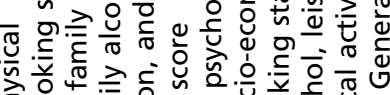

한 $\frac{0}{\frac{\pi}{0}}$

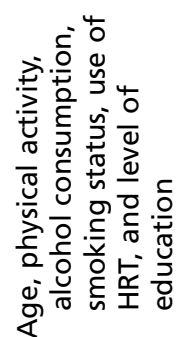

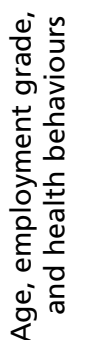

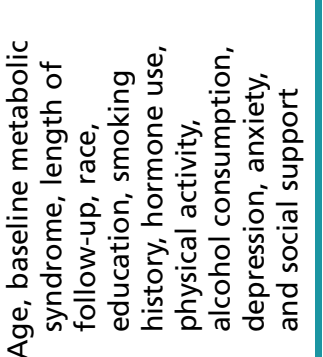
v

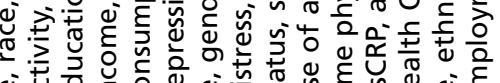

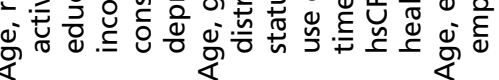

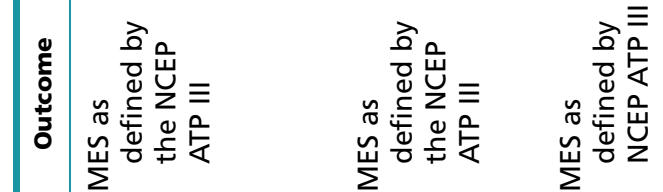

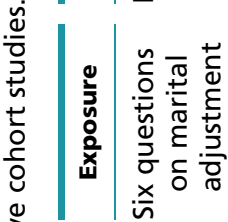

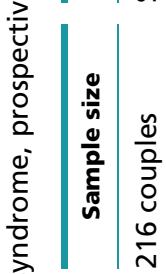
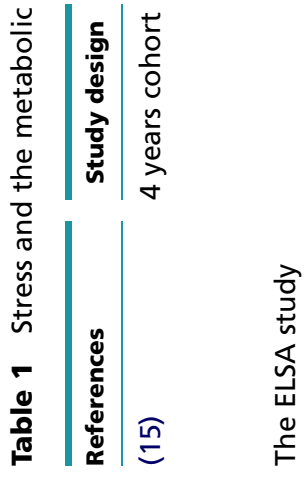

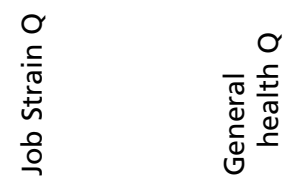

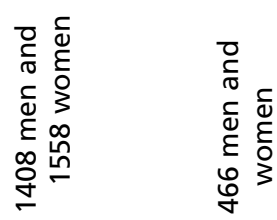

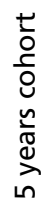

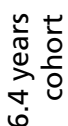

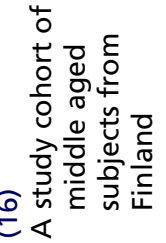

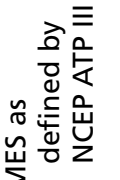

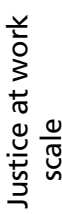

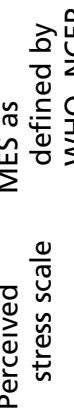

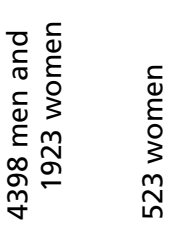

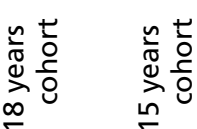

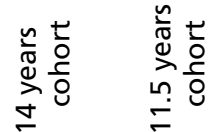

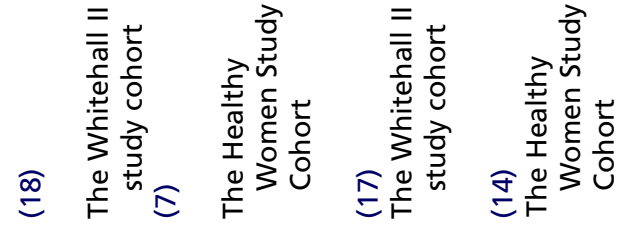

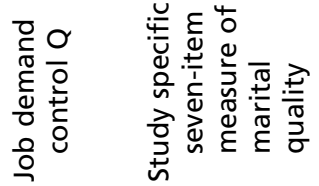

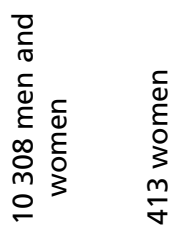

http://www.endocrineconnections.org DOI: 10.1530/EC-14-0031
() 2014 The authors Published by Bioscientifica Ltd

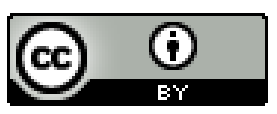

This work is licensed under a Creative Commons Attribution 3.0 Unported License. 


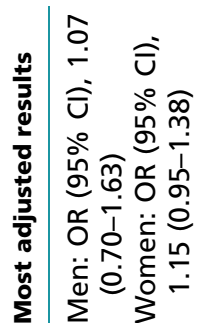

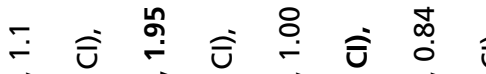

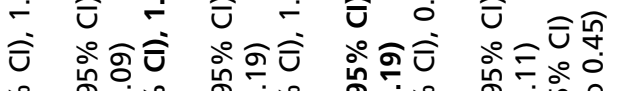

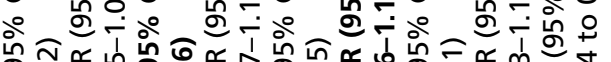

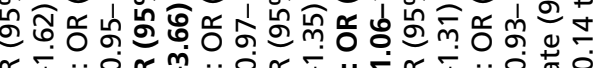

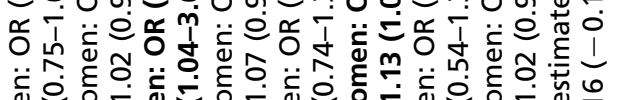

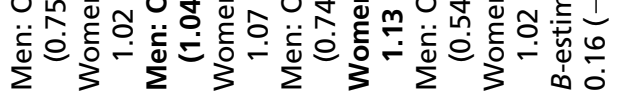

倇芒

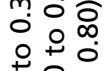

to

i1

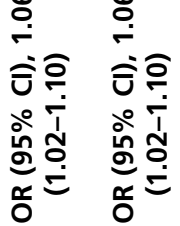

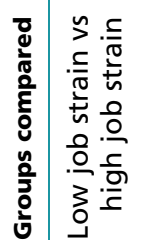

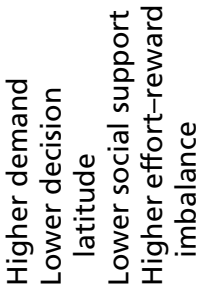

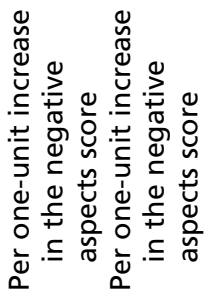
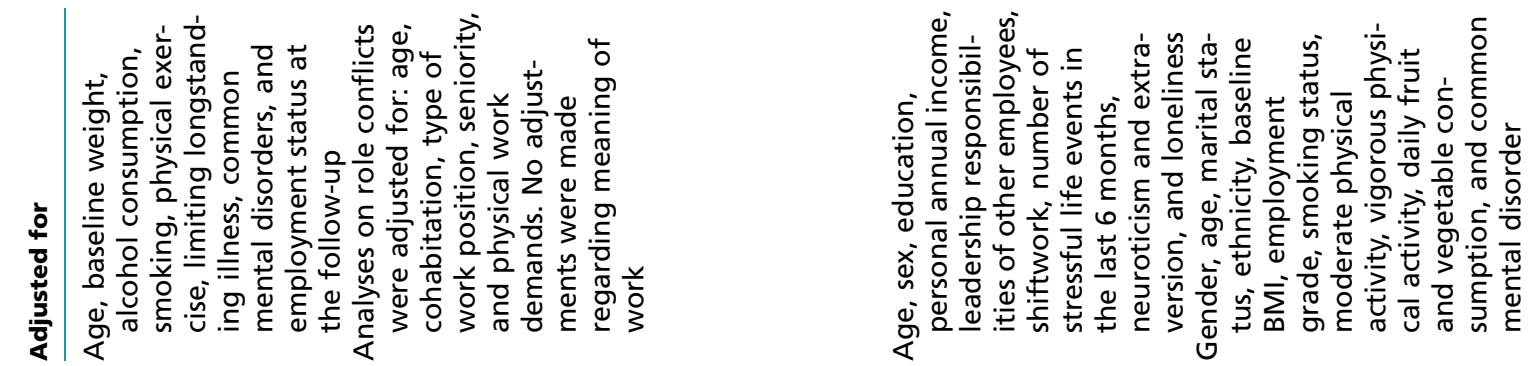

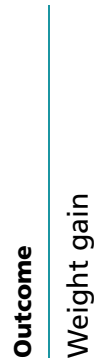

0
O
Oे

0
o
ò

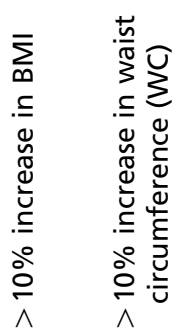

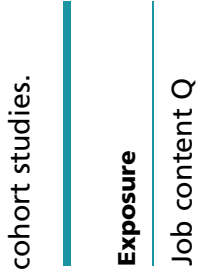

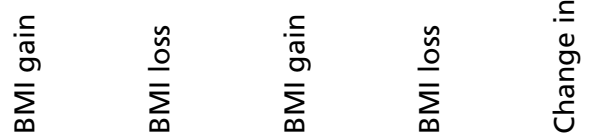

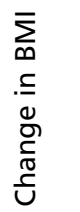

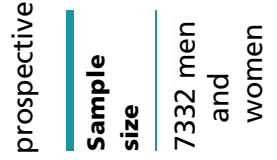

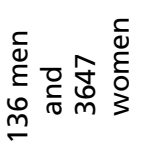

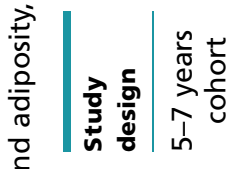

$\sum_{m}^{2} \frac{5}{0} \frac{5}{0}$

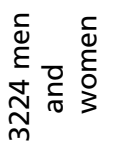

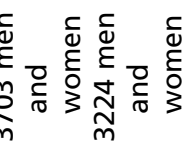

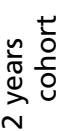

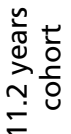

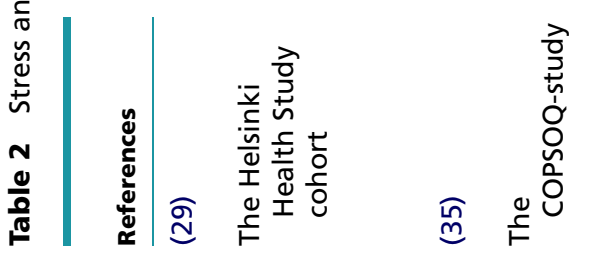

http://www.endocrineconnections.org

() 2014 The authors Published by Bioscientifica Ltd

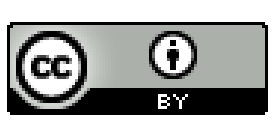

This work is licensed under a Creative Commons Attribution 3.0 Unported License. 


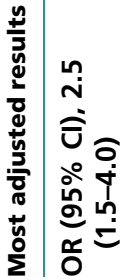

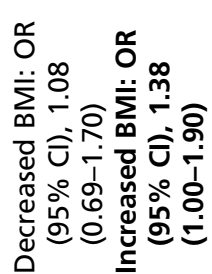

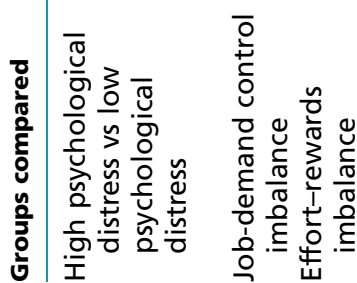

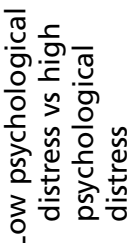

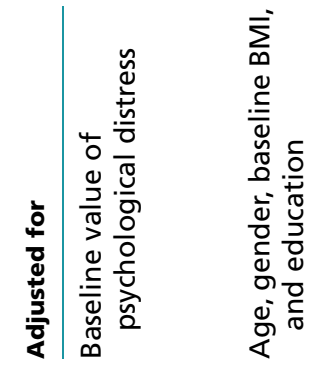

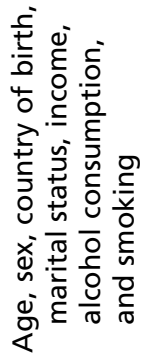

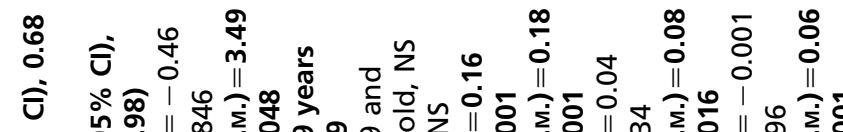

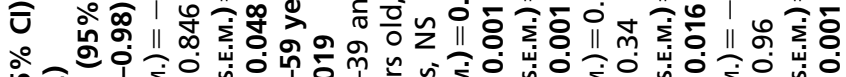

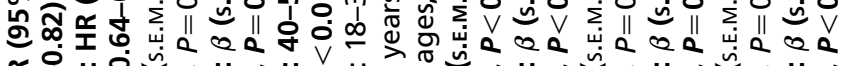

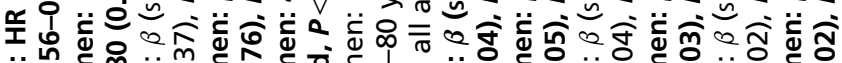

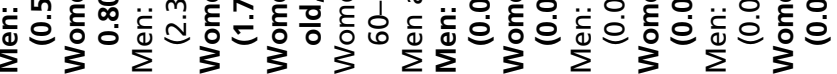

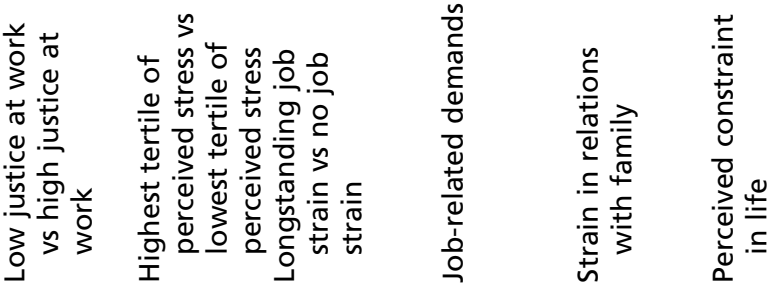

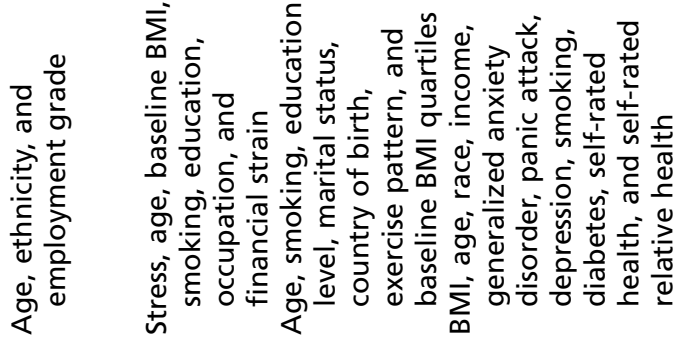
ᄒ

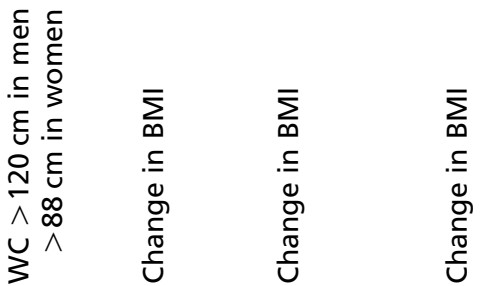

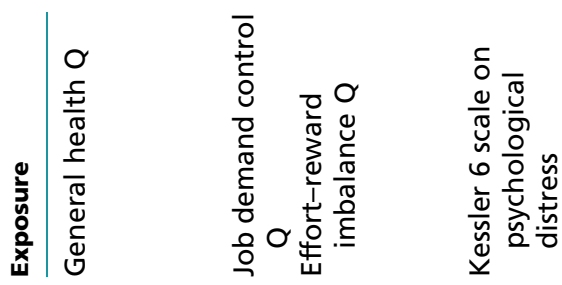

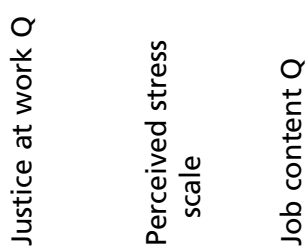

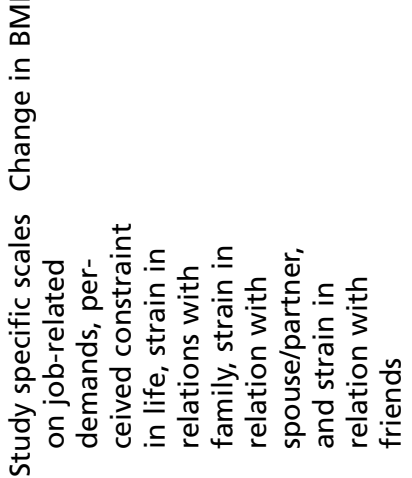

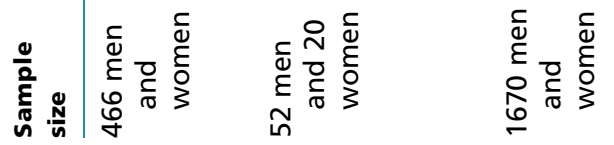

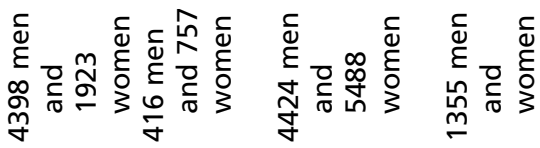

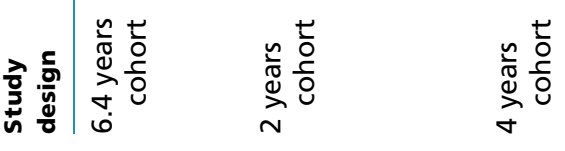

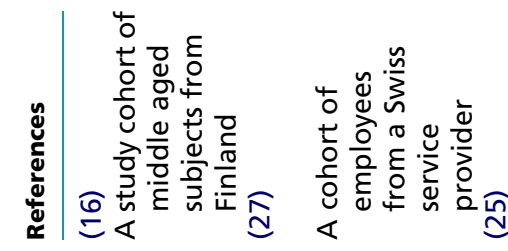

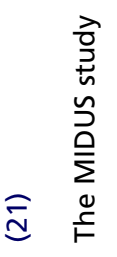




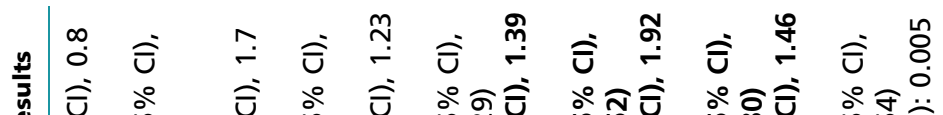

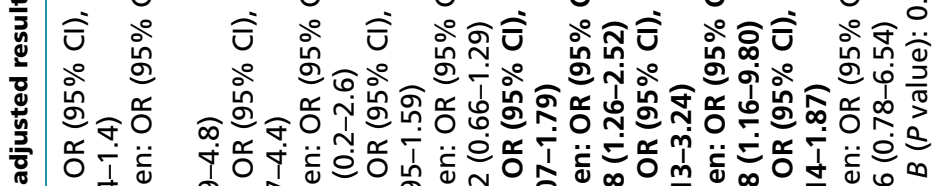

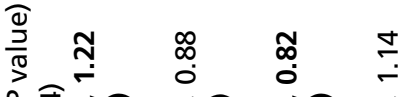

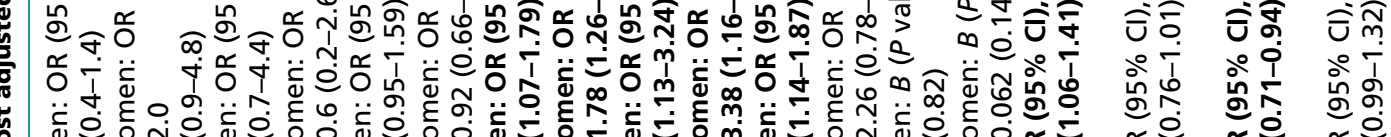

落
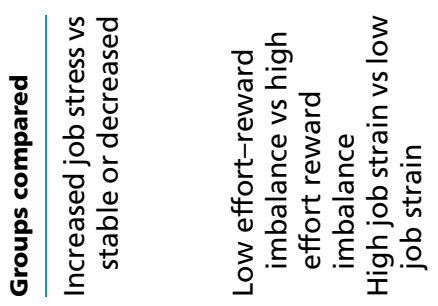

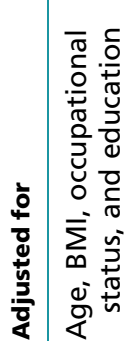

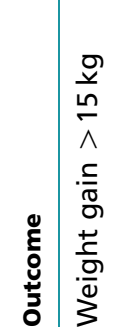

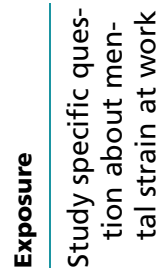

옹

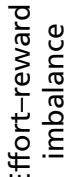

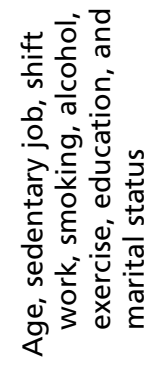

$\stackrel{\square}{ \pm}$

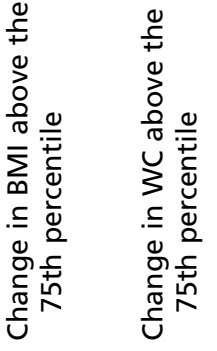

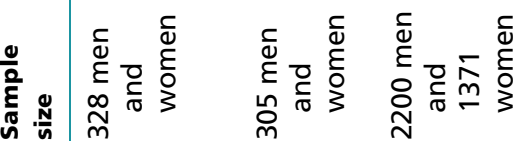

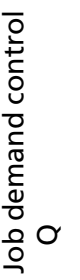

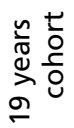

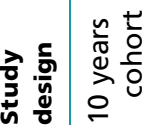

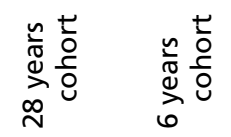

离

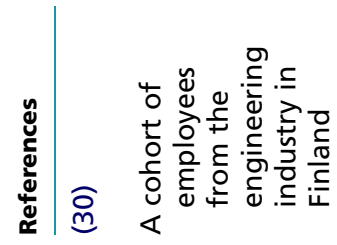

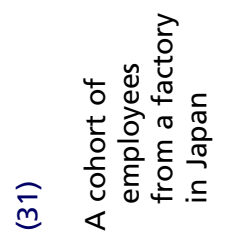

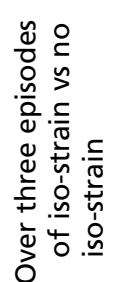
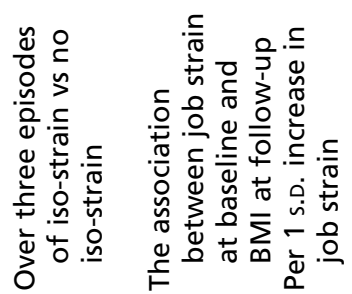

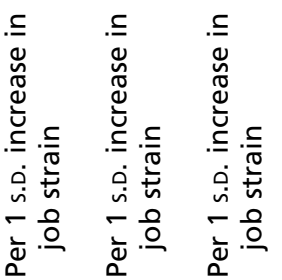

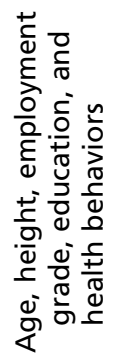

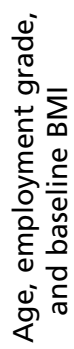
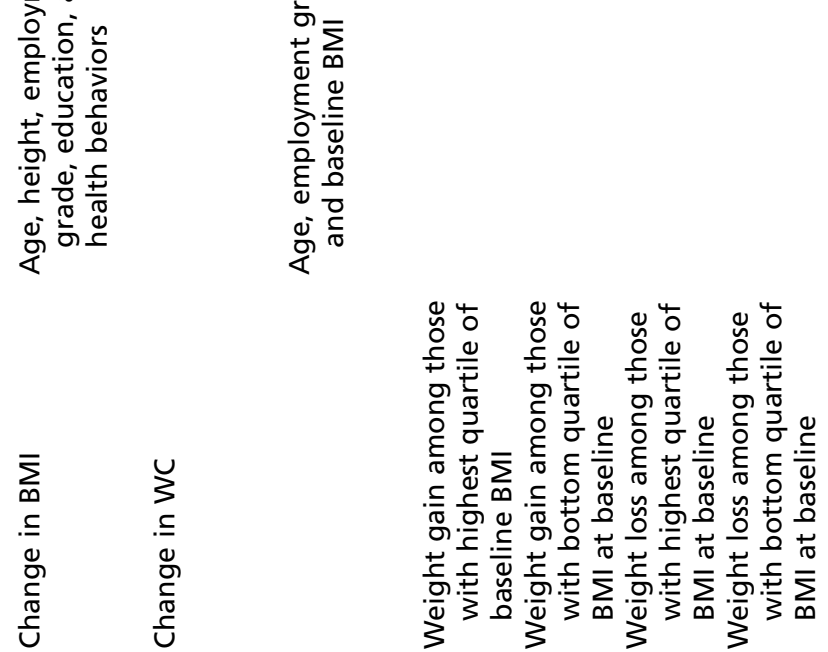
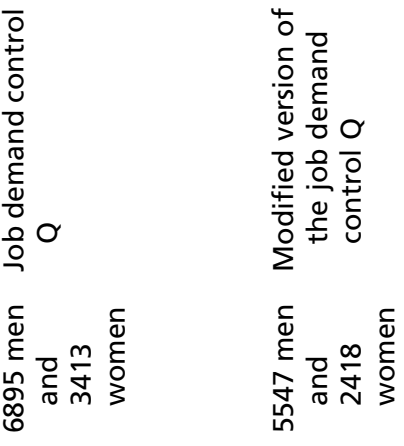

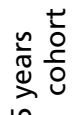
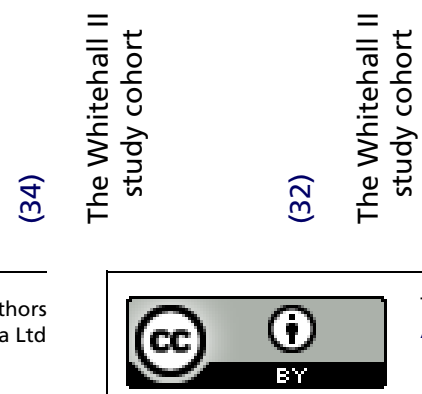

This work is licensed under a Creative Commons Attribution 3.0 Unported License. 

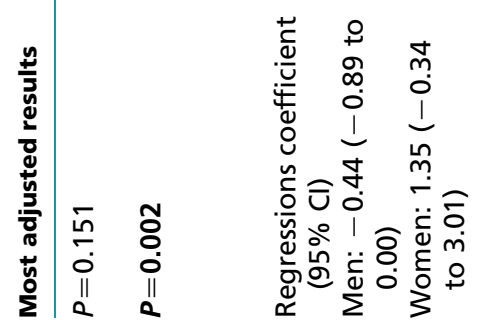

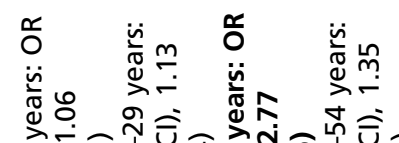

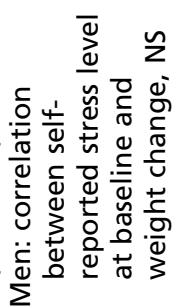

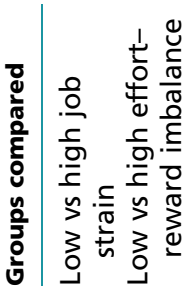

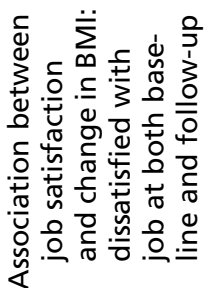

จิ

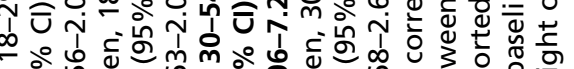

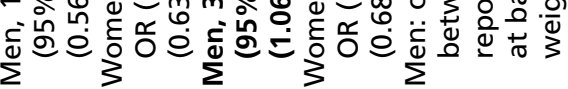
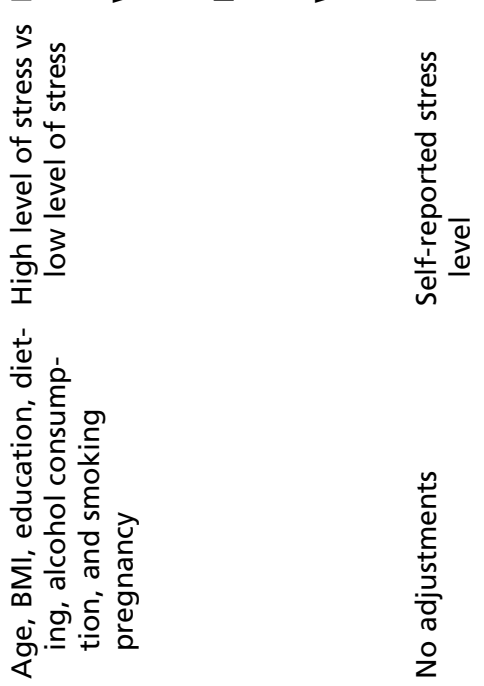

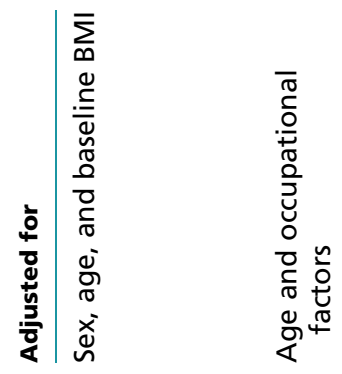

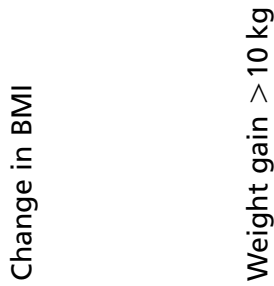

$\frac{0}{0}$
0
1
.$\frac{1}{\pi}$
$\frac{5}{0}$
$+\frac{1}{5}$
$\frac{0}{0}$
3

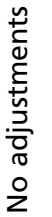

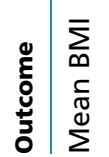

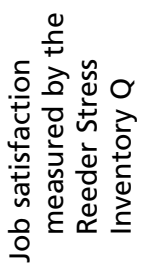

ธั

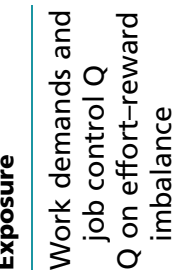

先 $\frac{\lambda}{\frac{\pi}{0}}$

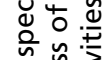

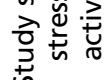

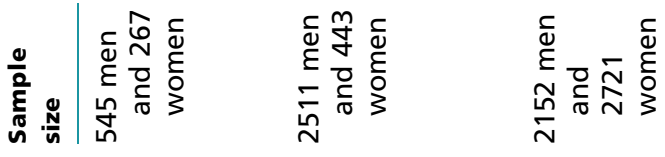

$\frac{5}{\pi}$
0
$\frac{1}{5}$
$\frac{0}{2}$
3

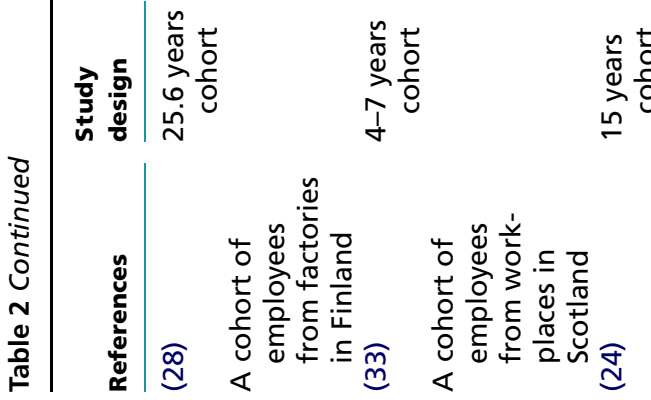

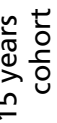

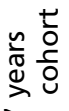



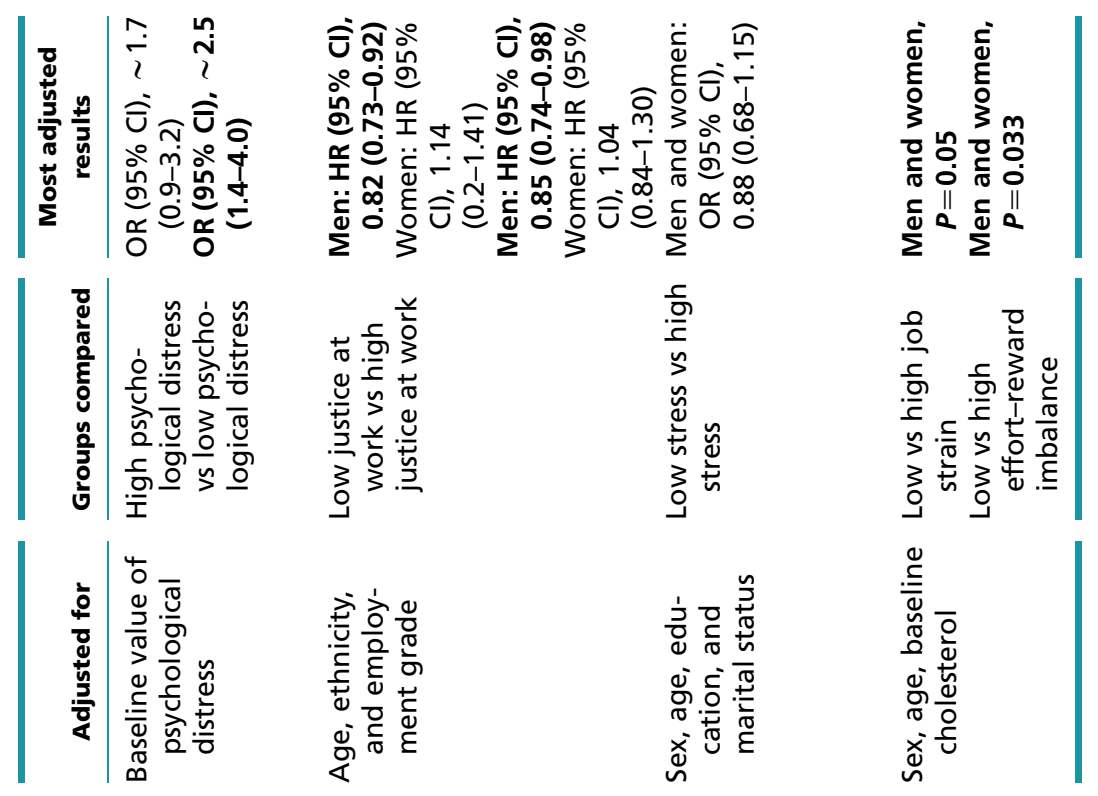

בั
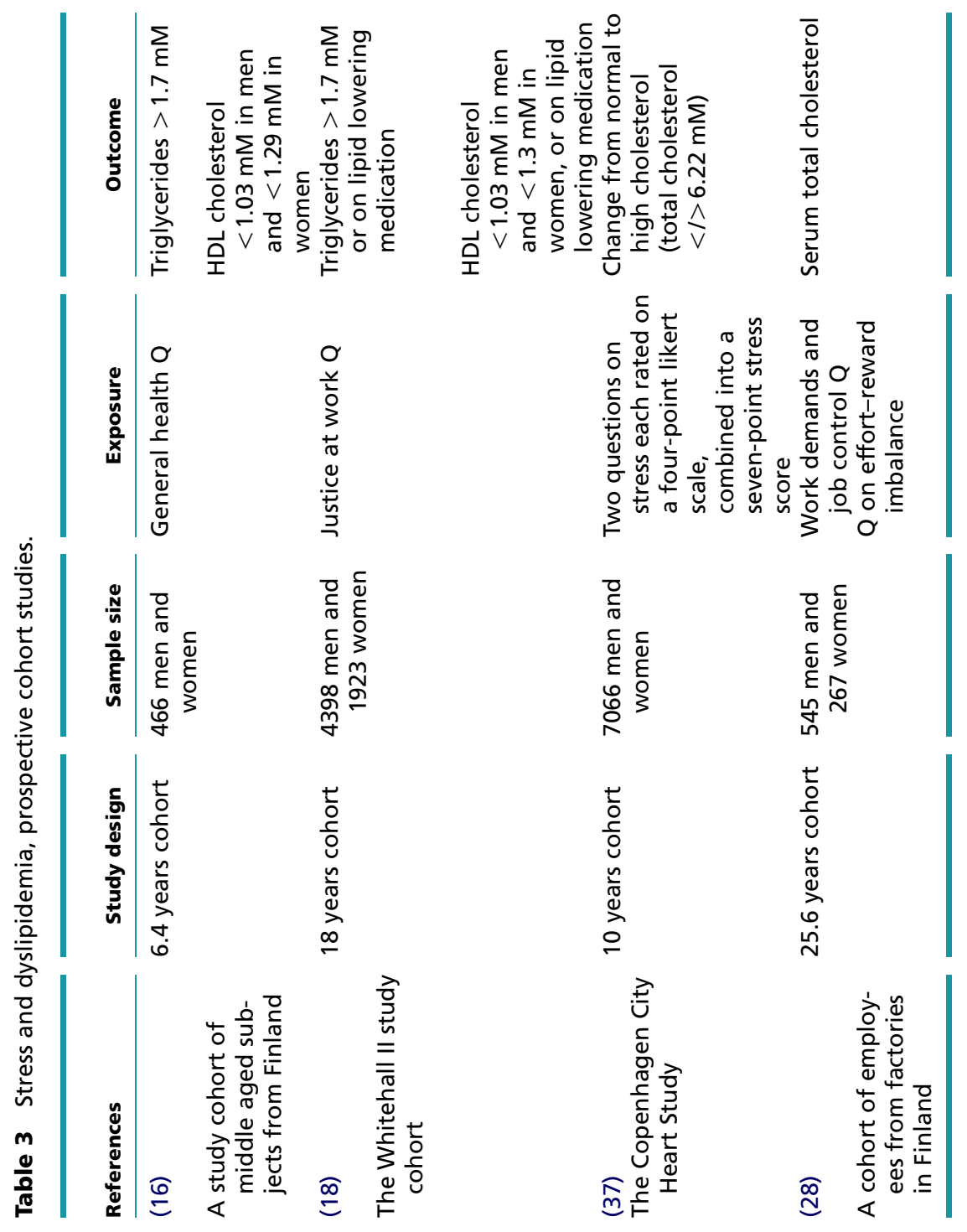

http://www.endocrineconnections.org

(c) 2014 The authors Published by Bioscientifica Ltd

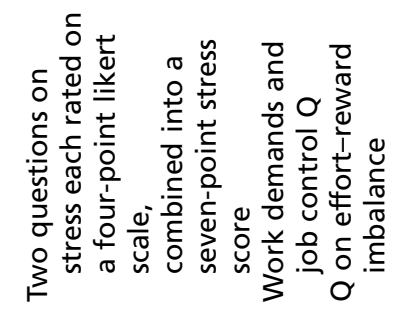

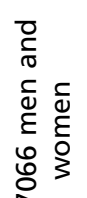

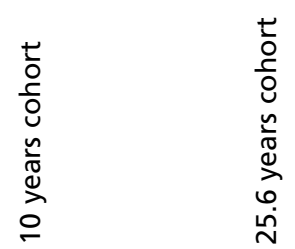

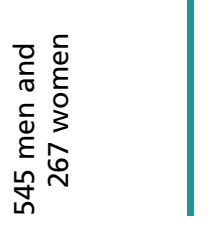

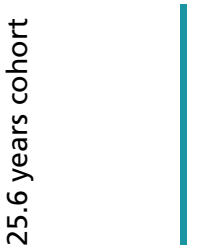

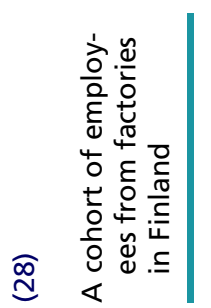

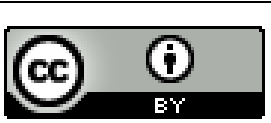

This work is licensed under a Creative Commons Attribution 3.0 Unported License. 


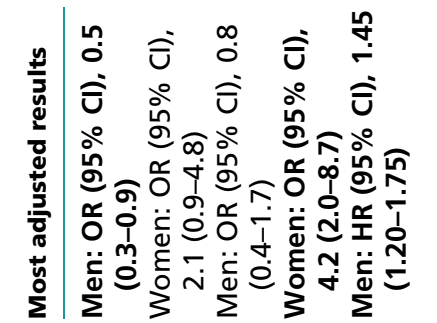

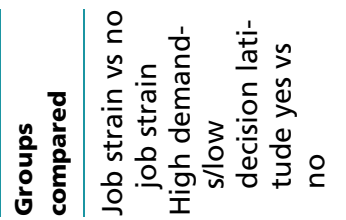

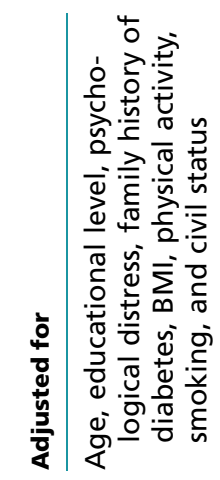

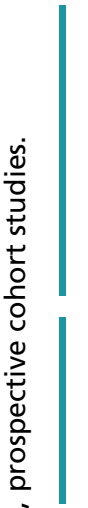

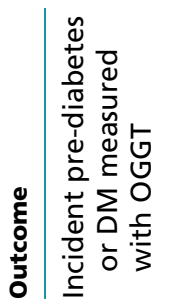

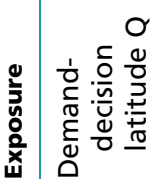

\}

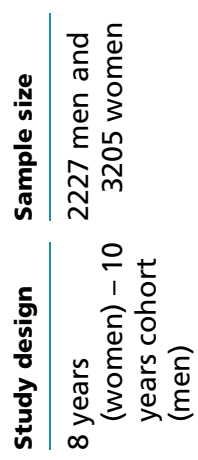

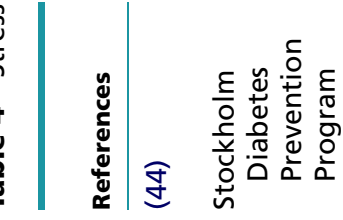

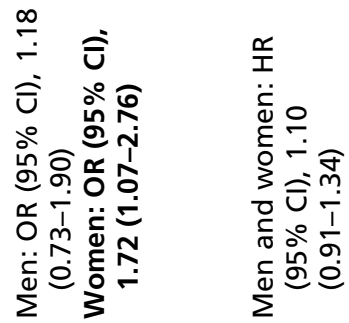

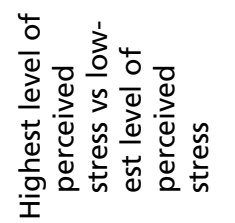
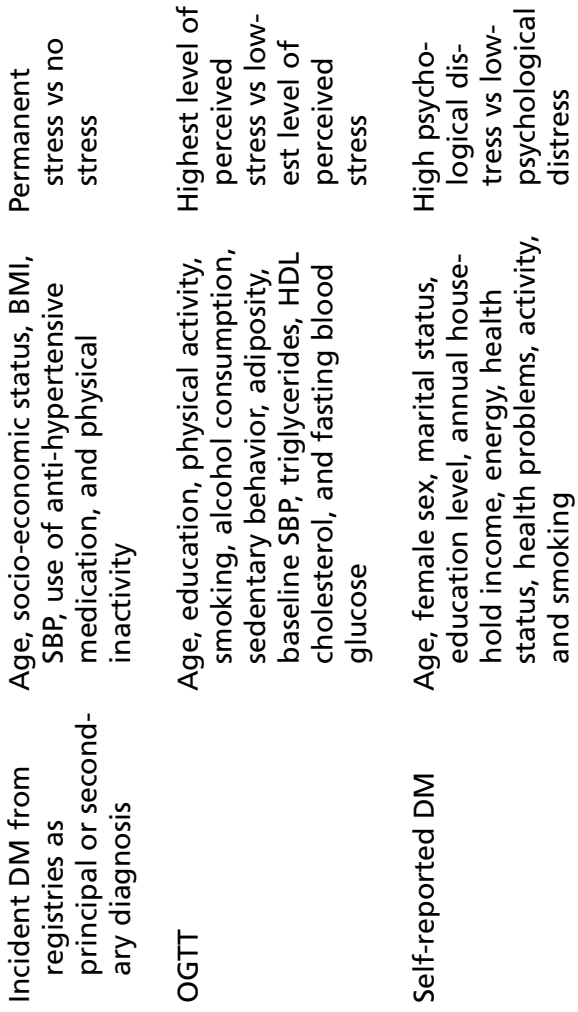

$\sum$
0
0
$\frac{1}{0}$
0
$\frac{0}{0}$
$\frac{4}{1}$
$\frac{1}{10}$
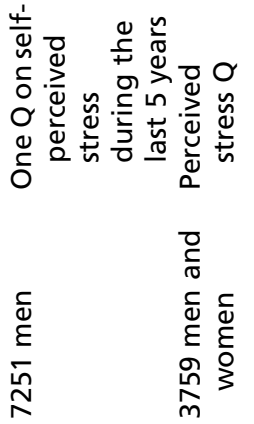

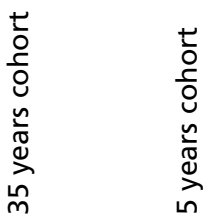

๙ู

ิิ

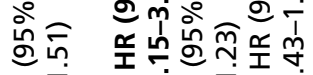

哇它

灾

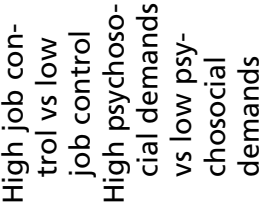

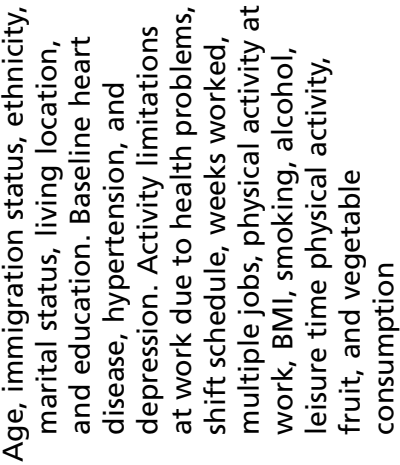

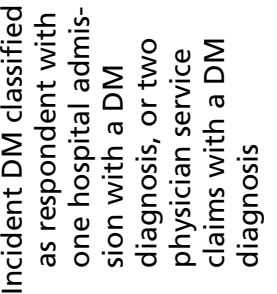

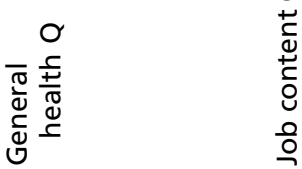

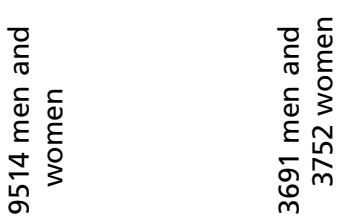

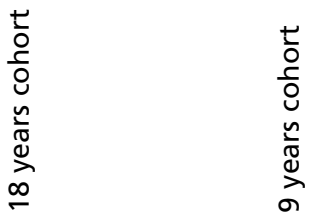

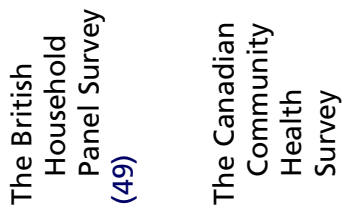

(c) 2014 The authors Published by Bioscientifica Ltd

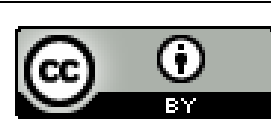




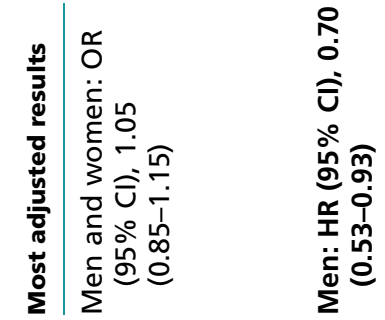

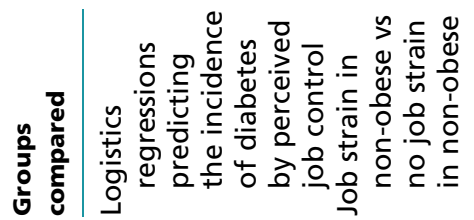
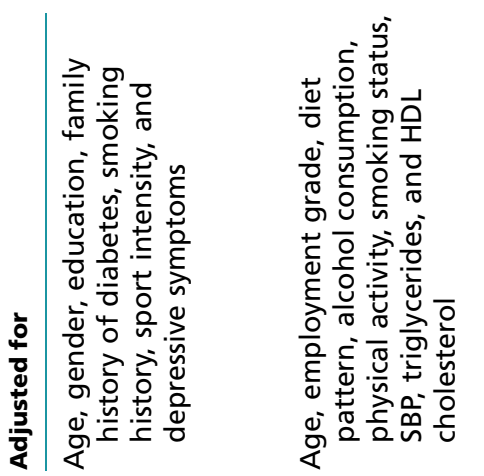

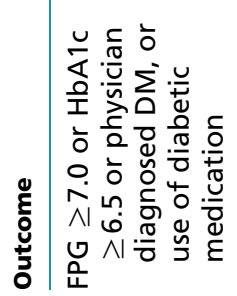

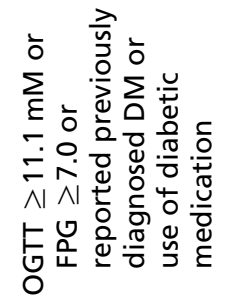

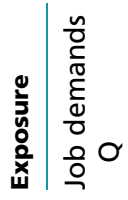

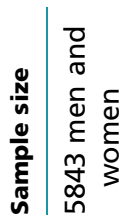

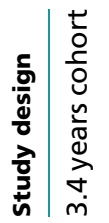

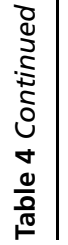

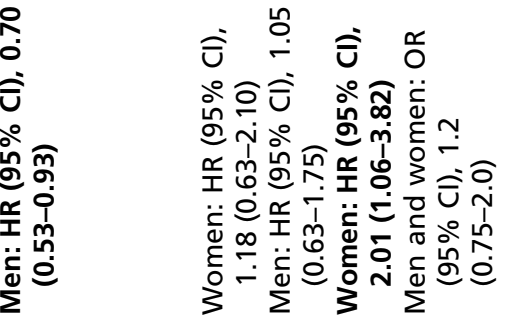

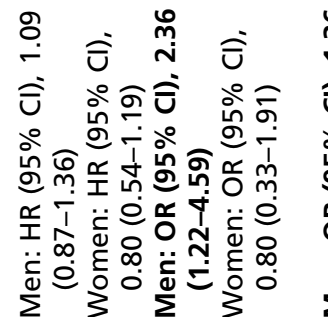

$\stackrel{\stackrel{m}{!}}{\dot{\mathrm{U}}}$

ธิ่

ผํำ

ơ

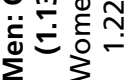

กั

茝

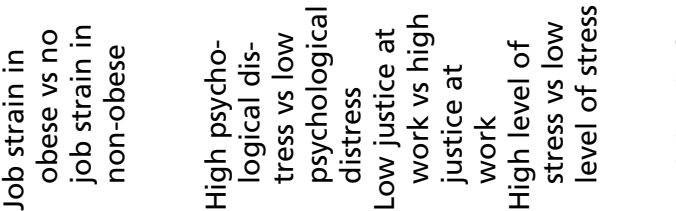

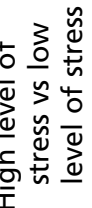

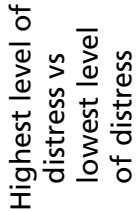

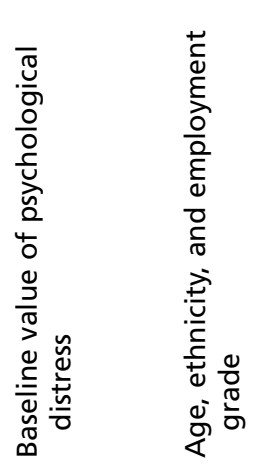

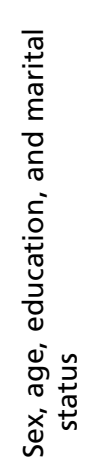

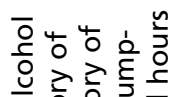

突

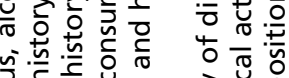

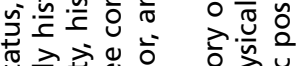

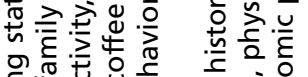

원.

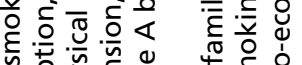

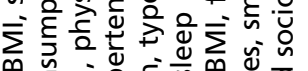

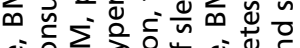

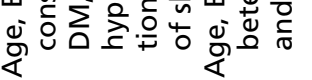

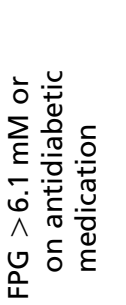

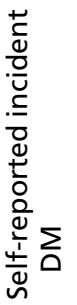

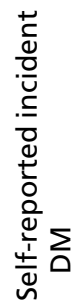

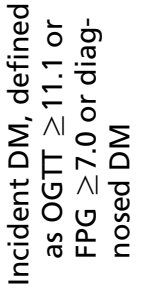

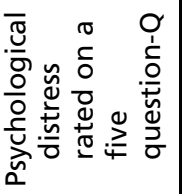

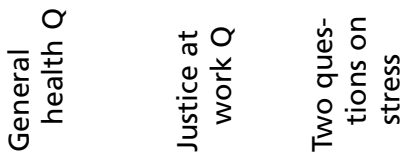

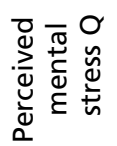

듐

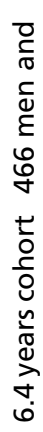

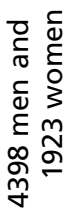

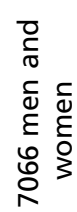

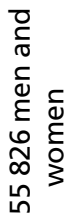

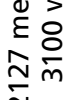

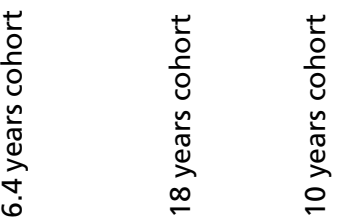

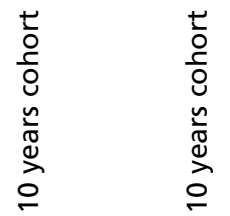

$\frac{\pi}{0}$
$\frac{0}{0}$
$\stackrel{0}{\sigma}$
$\stackrel{0}{0}$
0
0

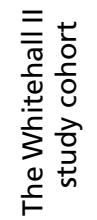

http://www.endocrineconnections.org DOI: 10.1530/EC-14-0031

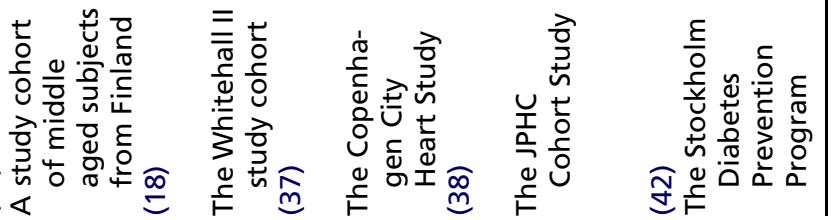

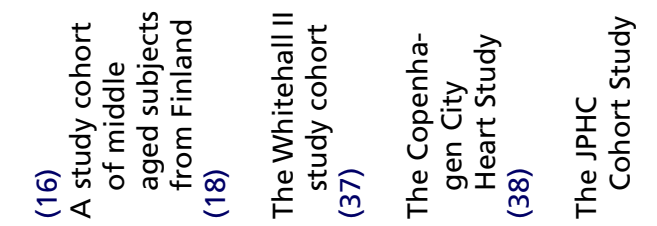

This work is licensed under a Creative Commons Attribution 3.0 Unported License. 


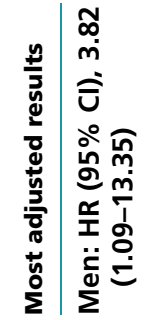

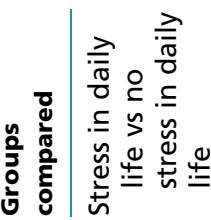

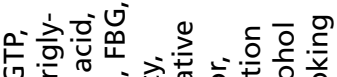

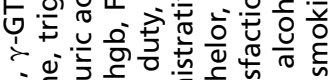

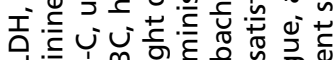

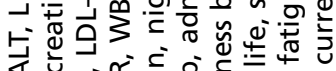

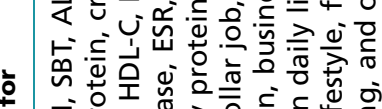

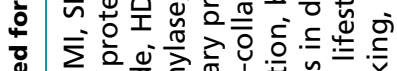

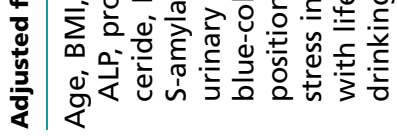

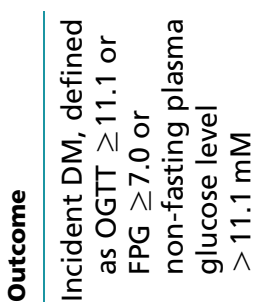

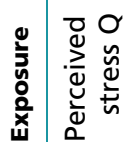

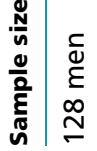

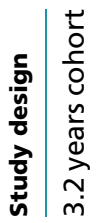

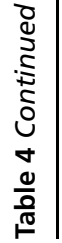

$\stackrel{\bar{\cup}}{ }$

ถึำ กิ่

$\stackrel{\propto}{\simeq}$

这导

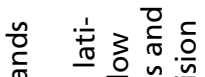

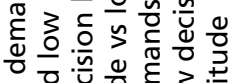

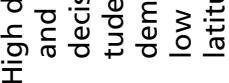

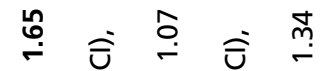

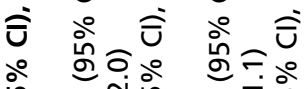

苞

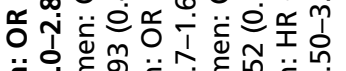

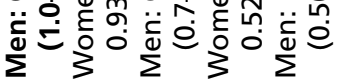

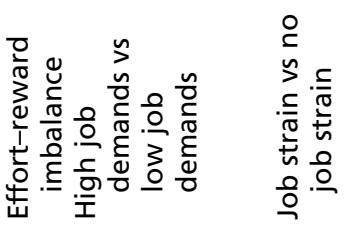

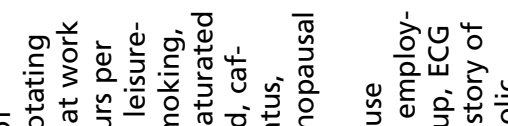

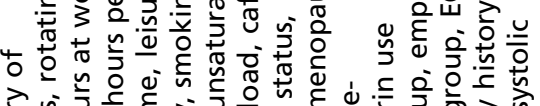

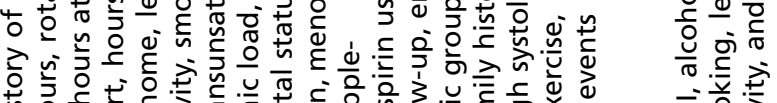

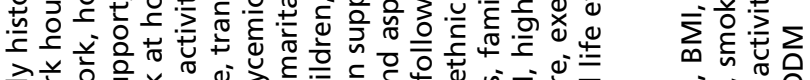

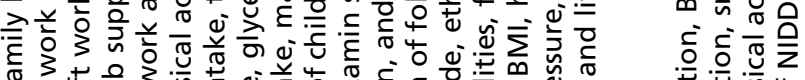

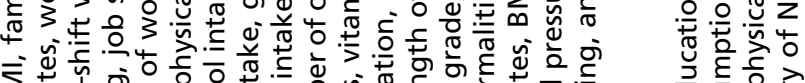

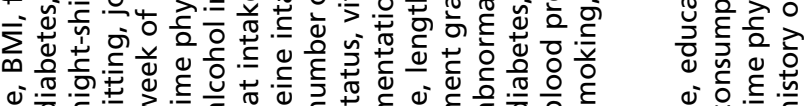

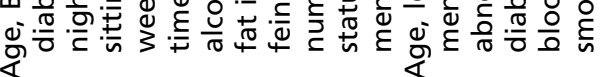
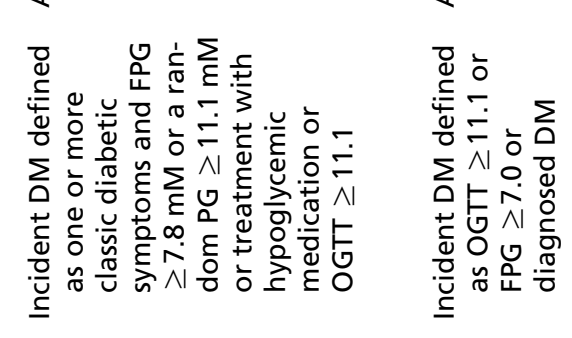

बं

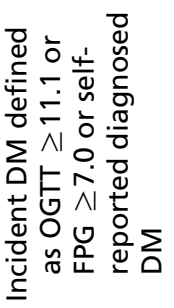

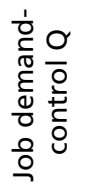

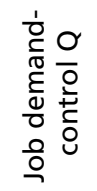

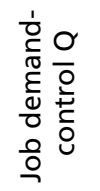

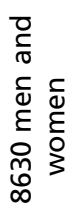<smiles>C1CCC(C2CCCC2)CC1</smiles>

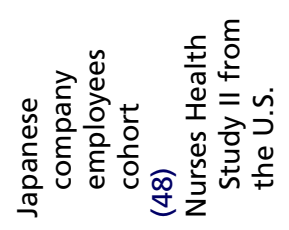

ֻัષ

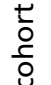

$\frac{5}{0}$

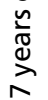

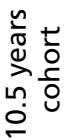

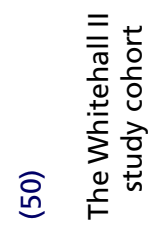



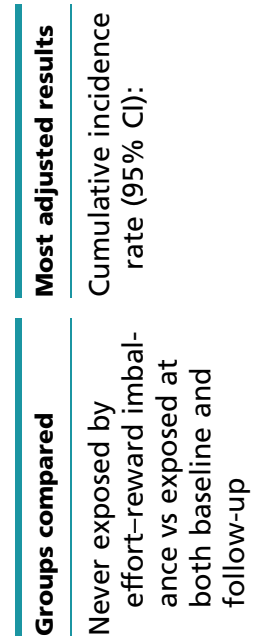

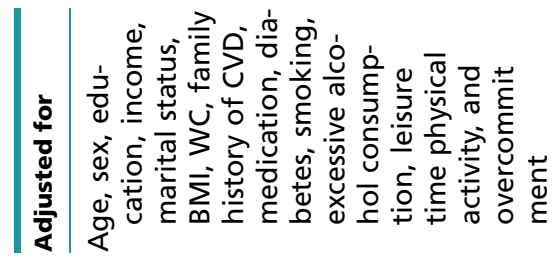
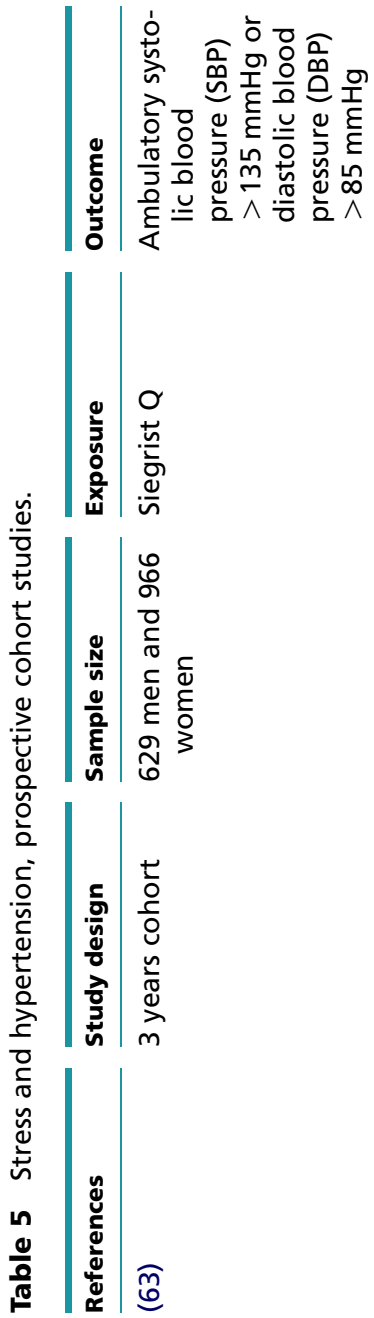
DOI: 10.1530/EC-14-0031
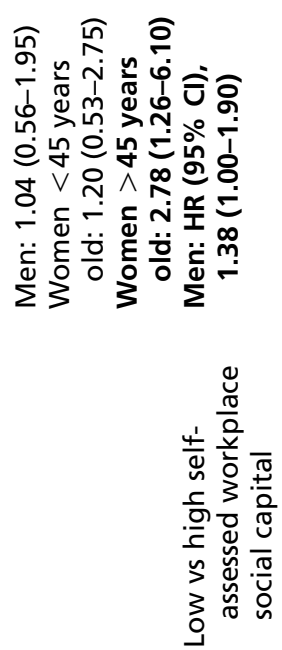

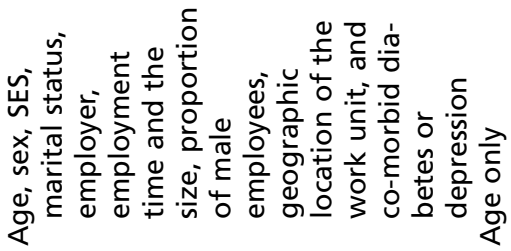
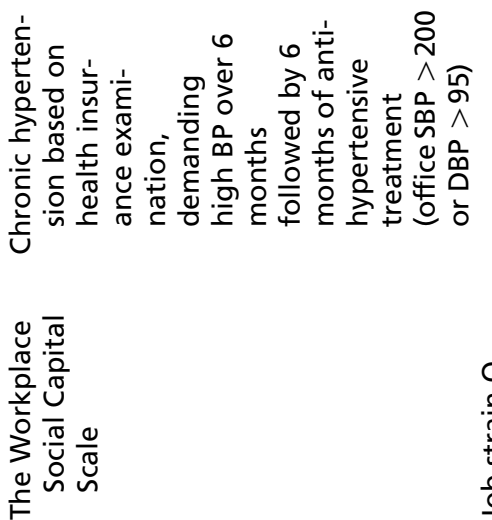

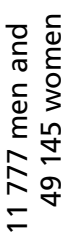

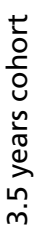

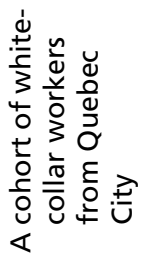

(C) 2014 The author Published by Bioscientifica Ltd

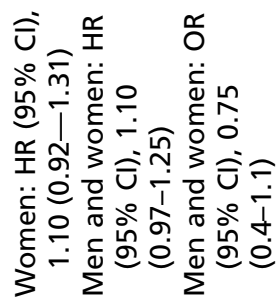

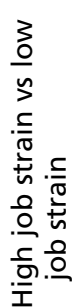
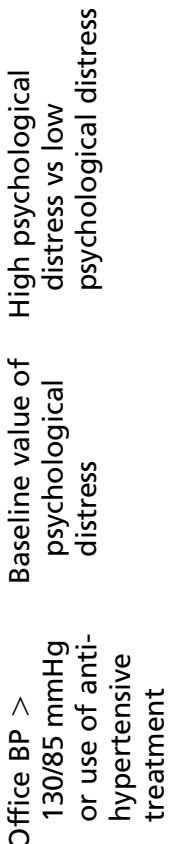

$O$
$\frac{1}{ \pm}$
$\frac{0}{0}$
$\frac{1}{\sigma}$
$\frac{0}{0}$
$\frac{1}{0}$

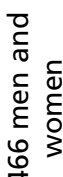

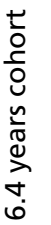

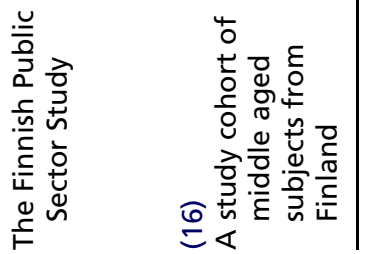



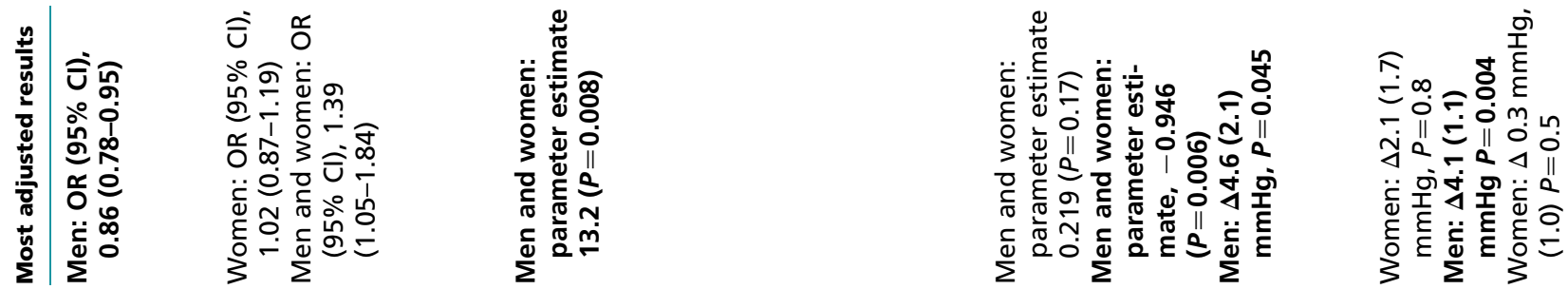

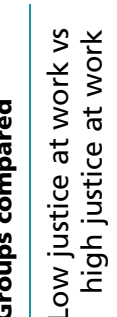

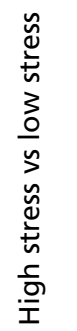

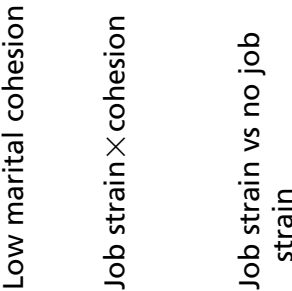

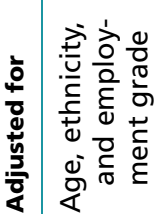

강 $\frac{\bar{\pi}}{\frac{\pi}{\frac{\pi}{2}}}$

음
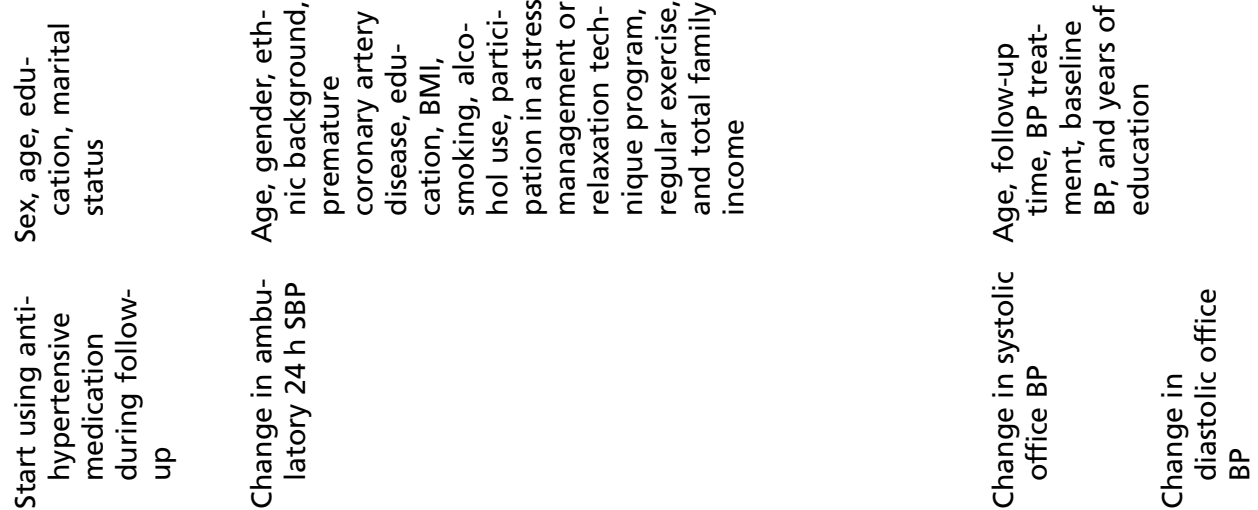

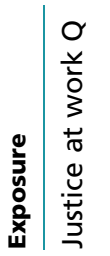

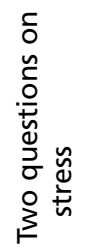

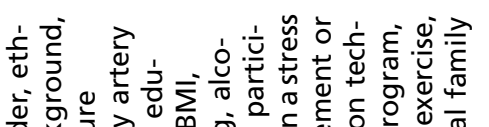

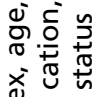

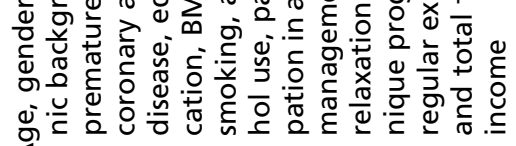

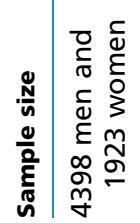

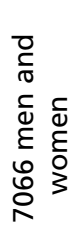

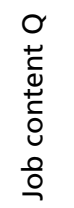

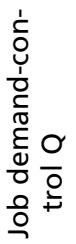

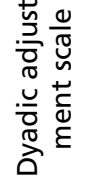

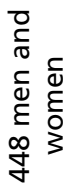

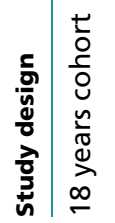

$t$
0
$\frac{1}{0}$
0
$\frac{0}{0}$
$\stackrel{1}{2}$
0

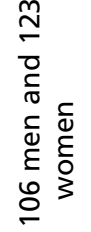

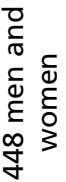
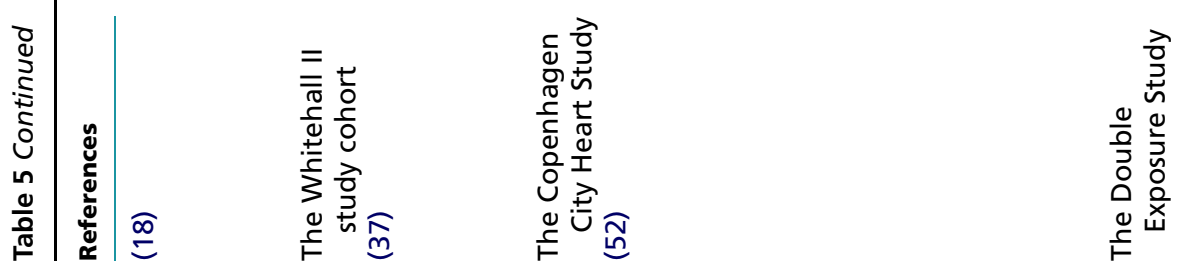

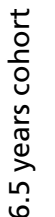

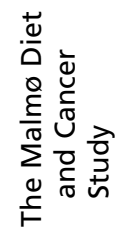




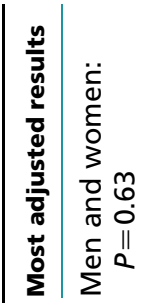

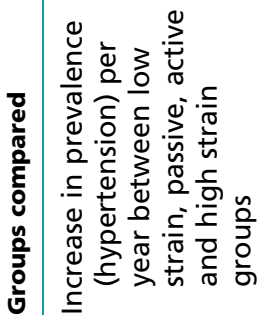

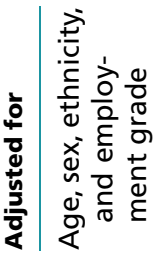

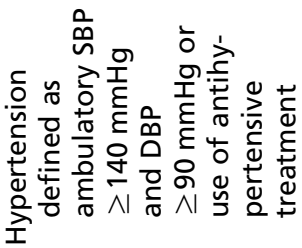

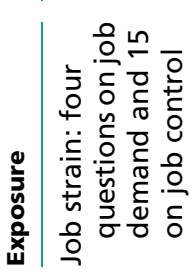

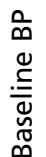

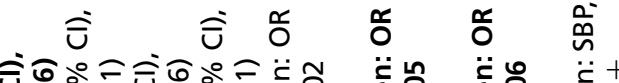

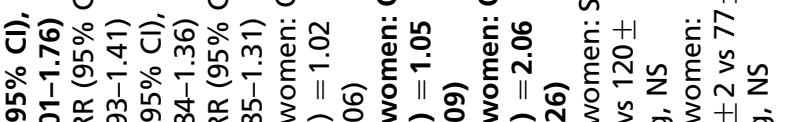

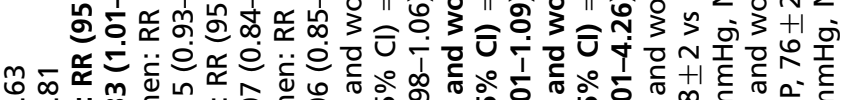

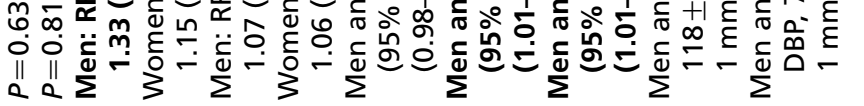

o

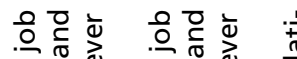

혼

党

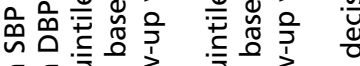

$\leq . \leq \bar{\sigma}$

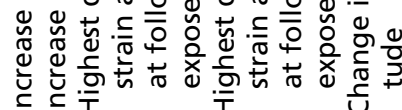

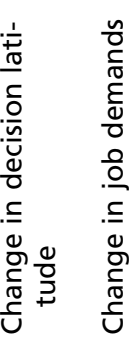

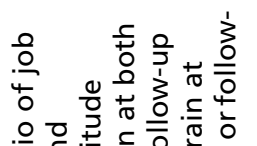

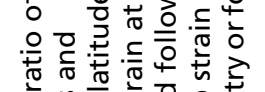

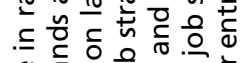

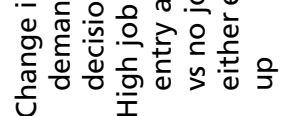

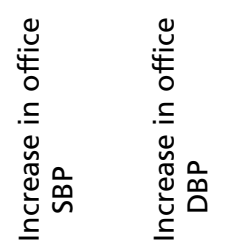

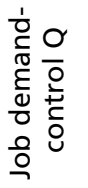

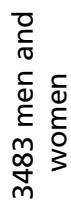

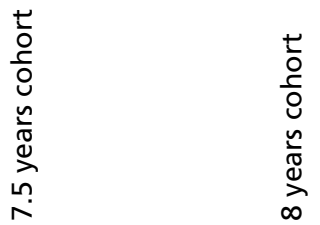

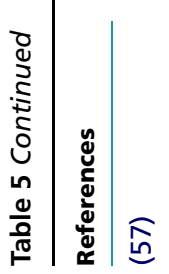

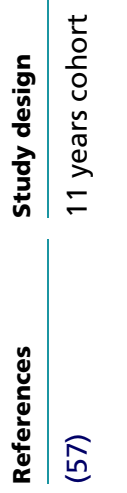

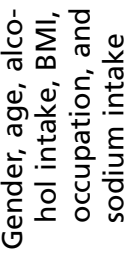

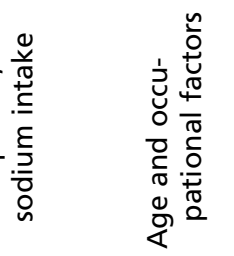

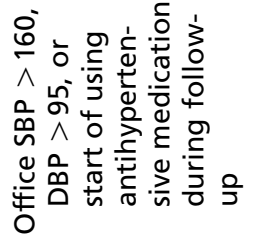

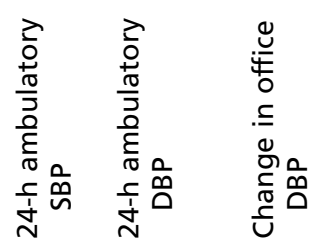

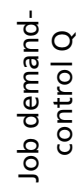

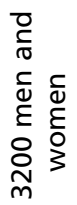

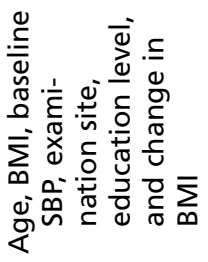

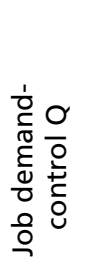

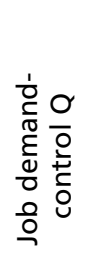

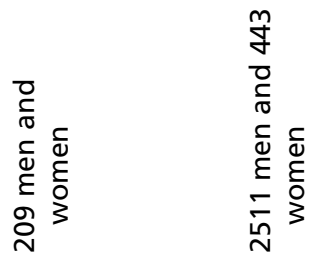

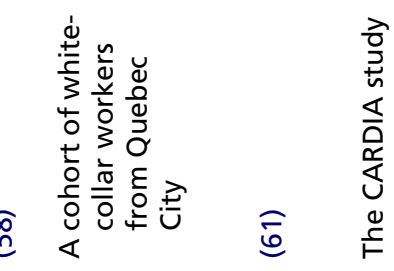

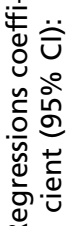

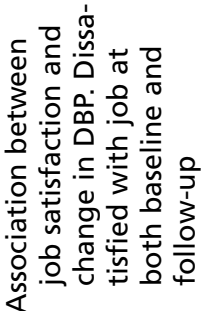

http://www.endocrineconnections.org DOI: 10.1530/EC-14-0031

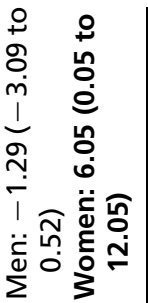

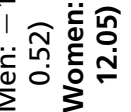




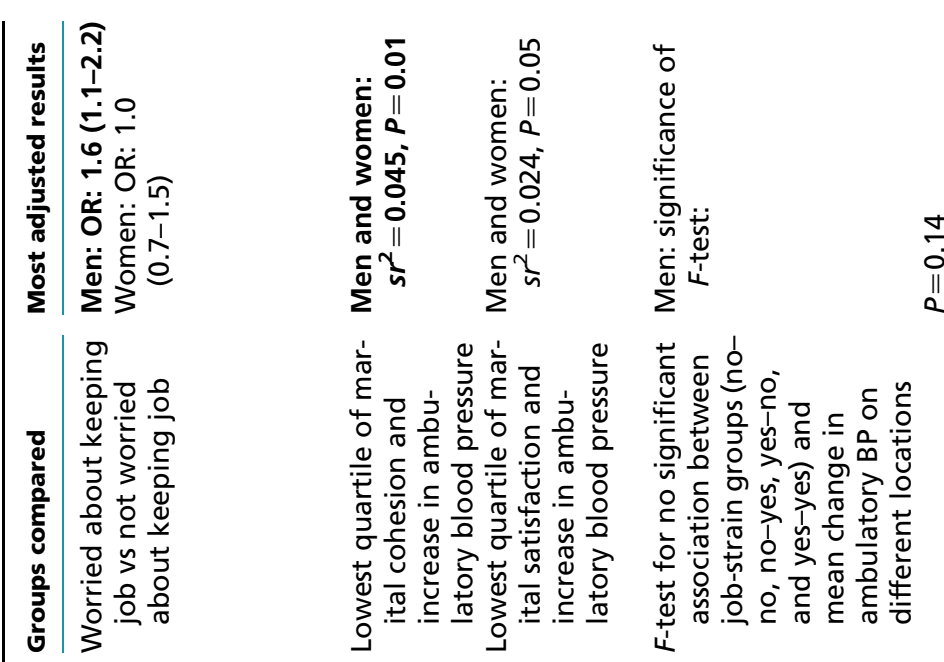

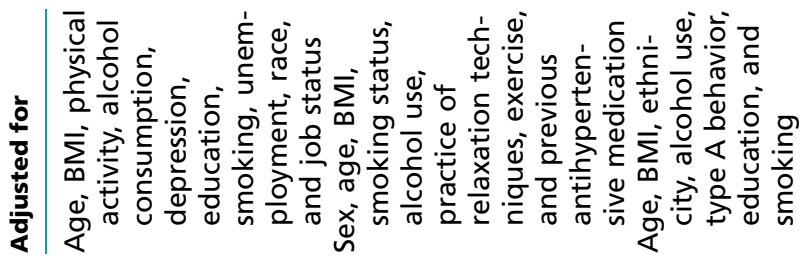
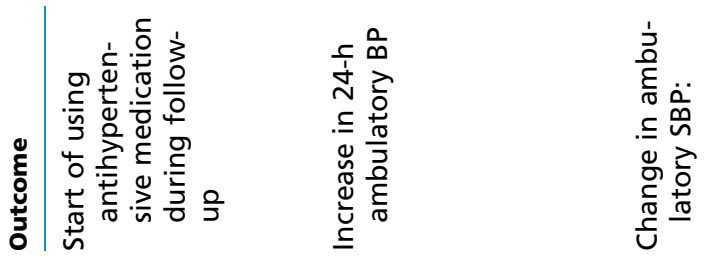

ํํำ

동응

II 000

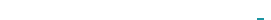

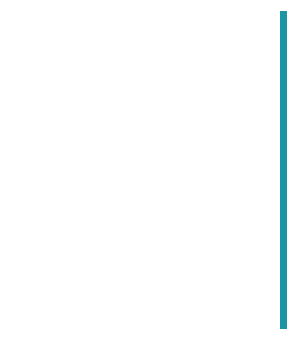

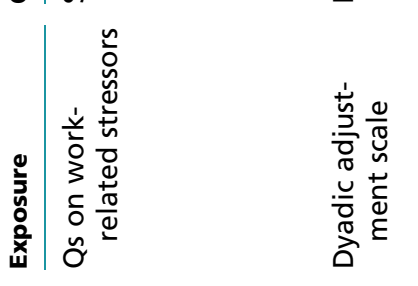

0
$\stackrel{1}{0}$
$\stackrel{0}{0}$
$\stackrel{0}{0}$
0
$\stackrel{0}{0}$
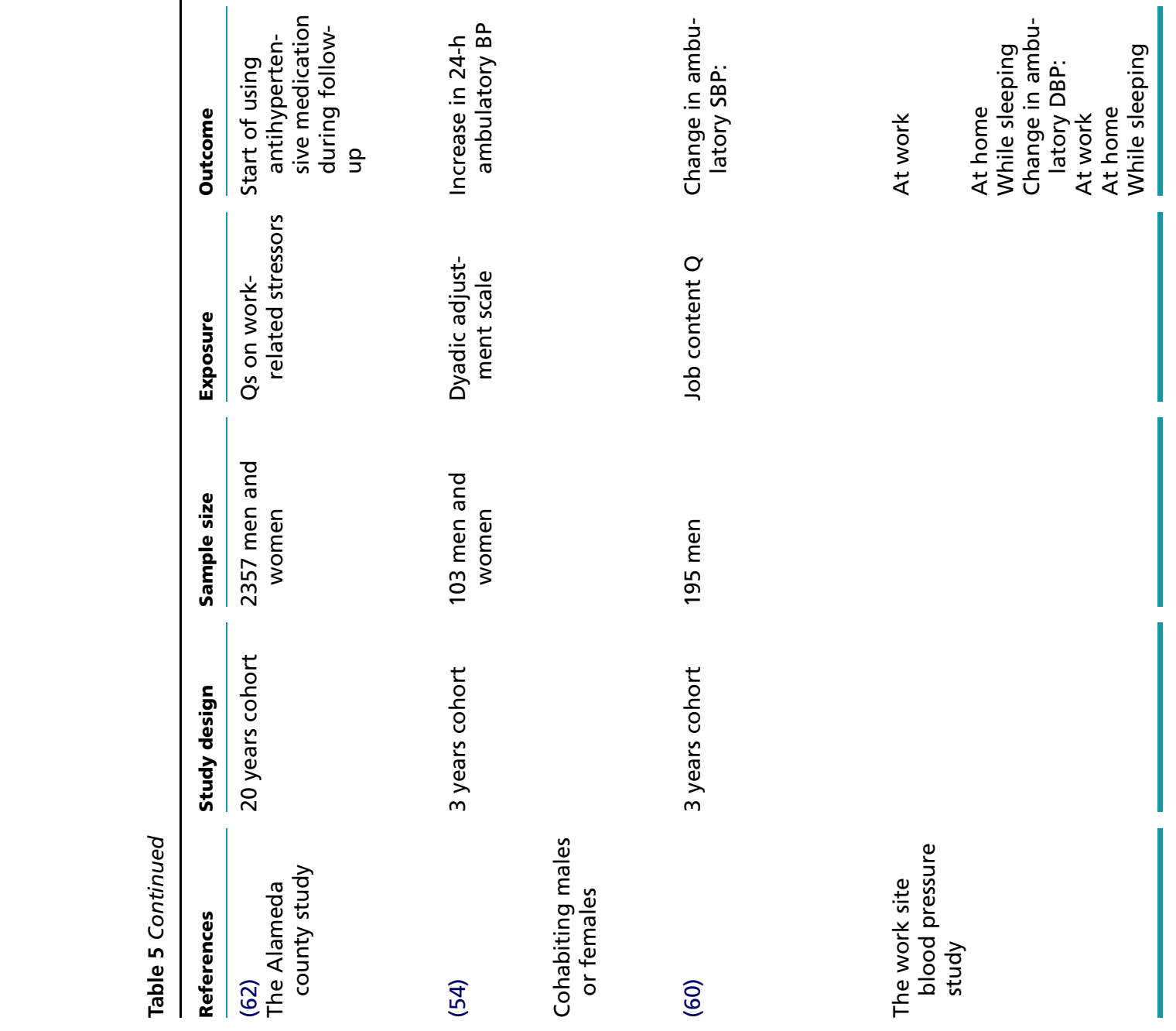

ป

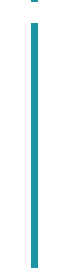

http://www.endocrineconnections.org

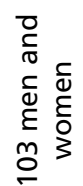

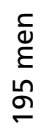

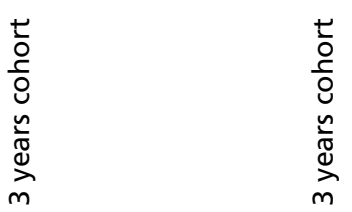
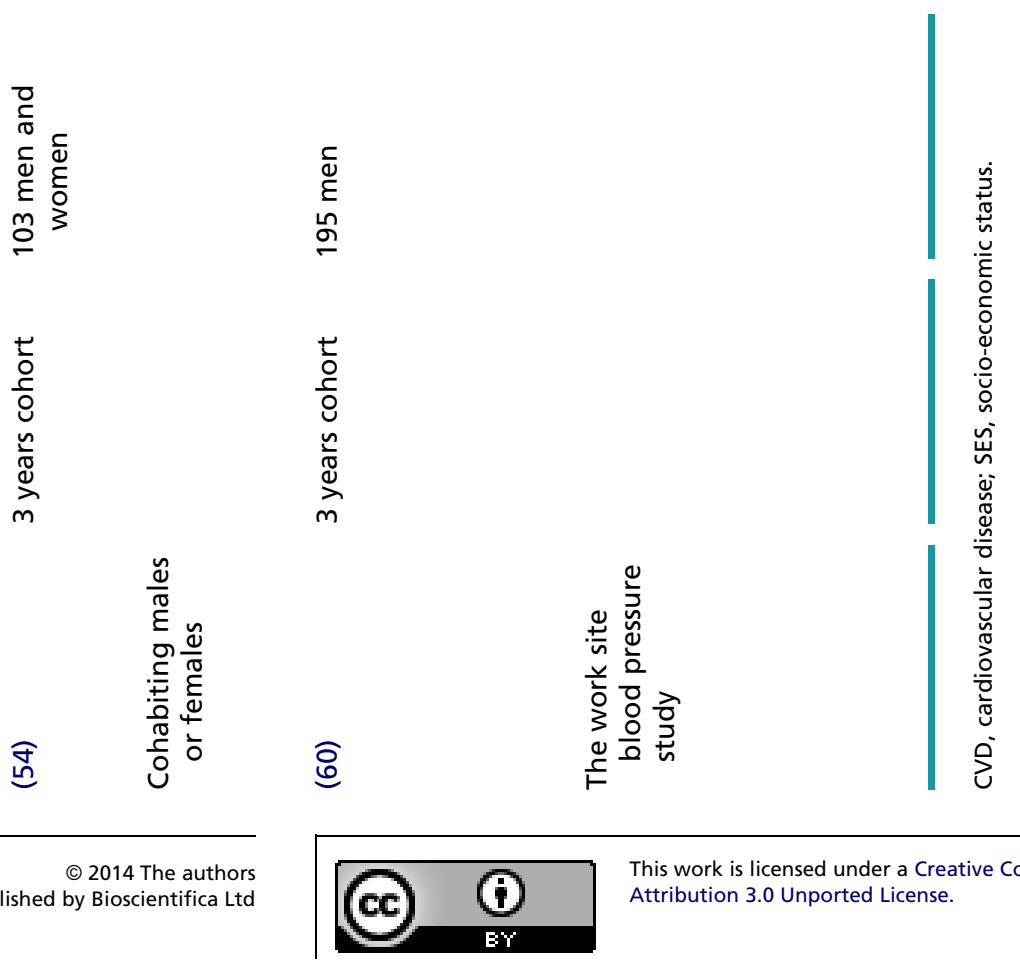

This work is licensed under a Creative Commons Attribution 3.0 Unported License. 
relationships, one evaluated strain in relations with family and perceived constraint in life, two studies evaluated self-perceived stress, one stress from daily activities, two evaluated distress and 13 studies on ten different cohorts evaluated WS.

As outcome variables four studies measured weight gain, three measured waist circumference (WC), ten measured BMI, and three measured both WC and BMI.

Relationship stress One study followed over 3000 men and women for 11 years and found that each unit increase in 'negative aspects of close relationship' score at baseline slightly increased the risk of a $10 \%$ or greater increase of weight gain at follow-up (20). The modest but significant effect was found both when outcome was measured as elevated BMI and as evaluated WC. The analyses were not separated by gender (20). The MIDUS study evaluated strain in relation with family among 1355 men and women and after 9.2 years of follow-up they found family strain to be a risk factor for weight gain among women but not among men (21).

Perceived stress The MIDUS study also evaluated perceived constraint in life and found a positive association between the level of constraints and weight gain among women, but not among men (21). This was supported by the Pitt County Study that found an association between perceived stress and change in BMI among women but not among men after 13 years of follow-up (22). Further a study including only men from one occupation (fireworkers) found no association between self-reported stress (measured by a nonstandardized Q) and weight gain after 7 years of follow-up (23). A study of the Finnish Twin Cohort separated the results both by gender and age, and found in contrast with the other studies that a high level of stress from daily activities was associated with a weight gain of over $10 \mathrm{~kg}$ in middle-aged men, but not among men of other age categories and not among women (24).

Distress A study of 466 men and women followed for 6.4 years found high psychological distress to increase the risk of weight gain measured as WC (16). This finding was supported by a study of 1670 men and women, which found psychological distress at baseline to be a predictor for increased BMI after 4 years of follow-up (25). Neither of the studies evaluated the results by gender.

Work stress Three of the 13 studies on WS did not separate their analyses by gender $(26,27,28)$. Two of these studies did not find a significant association between WS (effort-reward imbalance and job-demand control imbalance respectively) and increase in BMI $(26,27)$, while one study found effort-reward imbalance but not job strain to be significantly associated with change in BMI (28).

From the ten studies separating the analyses by gender, five did not support an association between WS and change in weight (measured as weight change or change in BMI) among either gender $(29,30,31,32,33)$, whereas two studies supported an association between BMI and WS among both genders $(21,34)$.

Concerning other measures of WS, a study evaluated the feeling of justice at work and found that a feeling of high justice was associated with a lower risk of a large WC ( $>120 \mathrm{~cm}$ for men and $>88 \mathrm{~cm}$ for women) after 18 years of follow-up among both men and women (18). A study on 3982 female and 152 male Danish health care workers evaluated other psychosocial work characteristics association with change in BMI and among other results they found role conflicts to be associated with a small increased risk of weight gain among women but not men (35).

Separating results by age Two studies separated their results by age and found age-dependent associations: one study found a high level of stress to be a predictor of major weight gain among middle-aged men (30-54 years old), but not among women and among younger age categories of any gender (24). Likewise, in a 5-year cohort of 4424 men and 5488 women, job strain was found to be significantly associated with change in BMI among middle-aged women, but not among women of other age categories and not among men (36).

Separating results by baseline BMI In a re-analysis of the Whitehall II cohort, subjects were stratified according to BMI at baseline (32). The study found that men with BMI $<22$ at baseline experienced a weight loss during WS, whereas men with a BMI $>27$ increase in weight $(P<0.006)(32)$.

BMI vs wc Three studies included both BMI and WC as outcome measures, and found that the association between stress and adiposity might be dependent on the outcome measure chosen: one study found job strain to be associated with increased BMI among both men and women, but found job strain to be associated with WC only among men (34). Another study found high job strain to be a risk factor for increased WC but not for BMI among both genders (31). While a study on the Whitehall II cohort, not separating the results by gender, found negative aspects of close relationships to be associated with both increase in WC and in BMI after 11 years (20).

This work is licensed under a Creative Commons Attribution 3.0 Unported License. 
Conclusion Studies regarding stress from relationships and overall perceived stress generally supported an association between stress and weight gain, especially among women. Distress seems supported as a risk factor for weight gain among both genders. Concerning the association between WS and weight gain, the studies found conflicting results, with approximately half of the studies finding no association, and the other half finding some association among either men or women. Differences have been found between studies measuring WC or BMI as outcome variables. One study found WS to have a bidirectional effect causing both weight gain and weight loss depending on baseline BMI, which might also contribute to the conflicting results found.

Stress and dyslipidemia - We included four studies (on four different cohorts) which prospectively examined the influence of chronic stress on blood lipids: one study focused on perceived stress, one on distress, and two studies focused on WS.

Perceived stress A study of 7066 men and women followed for 10 years found no association between perceived level of stress and lipid levels at neither baseline, nor at follow-up (37).

Distress One study found high psychological distress to be a risk factor for low HDL cholesterol levels after 6.4 years among 466 men and women (16). A tendency of a higher risk of elevated triglycerides was also found, although this was not statistically significant (16).

Work stress Concerning WS, one study followed 4398 men and 1923 women for 18 years and found that low justice at work was associated with the development of reduced HDL cholesterol and elevated triglycerides among men but not among women (18). Another study evaluated job strain and effort-reward imbalance among 545 men and 267 women and found high baseline job strain to be associated with a higher total cholesterol level after 25 years while no association was found regarding effortreward imbalance (28).

Conclusion Three out of four studies available supported some association between stress and the development of dyslipidemia. These limited data call for further studies in order to obtain a firm conclusion.

Stress and type 2 diabetes mellitus $~$ Sixteen prospective cohort studies, with type 2 diabetes mellitus (DM2) as outcome variable, were included. Five studies evaluated the exposure of perceived stress, three evaluated distress, while eight studies focused on WS. The studies focusing on other parameters than WS were all based on different cohorts. Three of the included studies on WS were based on data from the Whitehall II cohort, while the remaining five studies were based on different cohorts.

Perceived stress In a study of 7066 participants men but not women, with a high level of general stress had more than two-times increased risk of developing DM2 during 10 years follow-up (37). This was supported by a study of 55826 men and women, which found that men, but not women, with a high level of perceived mental stress had a 1.4 higher odds of developing DM2 during 10 years of follow-up as compared with men with a low stress level (38). A higher risk of incident DM2 after 3 years of follow-up was also found among male Japanese employees rating a high level of stress in daily life as compared with employees rating no stress in daily life (39). Likewise, a study of 7251 men showed that self-perceived permanent stress (during the last 5 years) increased the risk of incident DM2, with a hazard ratio of 1.4 after 35 years of follow-up (40). However one study on 3759 Australian men and women did not find an association between high level of perceived stress and development of abnormal glucose tolerance (measured by oral glucose tolerance test (OGTT)) among men, but did find an association among women (41).

Distress One study included 2127 men and 3100 women and found that, after 10 years, men, but not women, with a high level of distress had an increased risk of developing DM2, as compared with persons with low level of distress (42). Another study measuring distress among 9514 men and women found an association between psychological distress and self-reported incident DM after 18 years of follow-up not separating the results by gender (43). However, the association became nonsignificant after controlling for socioeconomic status, e.g. educational level and income, and health status (43). A study on 466 men and women evaluating distress did not support an association between distress level and risk of having fasting glucose $\geq 5.6 \mathrm{mM}$ after 6.5 years of follow-up (16).

Work stress A study on middle-aged Swedish men and women found job strain to be associated with a decreased risk of DM2 among 2227 men after 8-10 years of followup, while no significant association was found among the 3205 included women (44), although this has been disputed recently by Brunner \& Kivimaki (45).

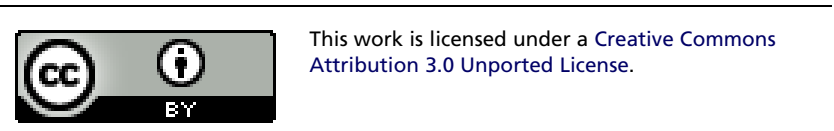


Nevertheless, this suggested protective effect of job strain found among men has also been found in a subgroup analysis of the Whitehall II cohort (46): when stratifying the results by baseline BMI, WS was found to be associated with a lower risk of developing DM2 among men with BMI $<30$, but a higher risk of DM2 among women with BMI $>$ 30 (46). No association was found between WS and DM2 among obese men and nonobese women and no association was found when the cohort was not stratified by BMI (46). Another study on 2597 Japanese male company employees found no association in either direction between job strain and the risk of developing DM2 after 8 years of follow-up (47).

With regard to control-demands imbalance the above-mentioned study on middle-aged Swedish men and women also separated WS into high demands/low decision latitude (44). In the study, they found no association between high demands and low decision latitude and DM2 among men, while in women it was associated with a four times increased risk of DM2 (44). In contrast, the Nurses Health Study found no significant association between high demands/low decision latitude and incident DM2 after 5.7 years of follow-up on 62574 women (48). Regarding job control, another study found a significant association between low job control and development of DM2 among women but not among men after 9 years of follow-up (49). The same study found no association between job demands and incident DM2 among either gender (49). Likewise, a study on 8630 men and women from the Whitehall II cohort found no association between high job demands and DM2 among neither men nor women after 10 years (50). A study of 5843 men and 1449 women found no significant association between job control and DM2, however, not stratifying their results by gender (51).

From the Whitehall II study cohort, data on justice at work and effort-reward imbalance were also evaluated: no association was found between level of justice and incident DM2 after 18 years of follow-up (18). In contrast effort-reward imbalance was found to be a risk factor among men but not among women after 10 years of follow-up (50).

Conclusion Perceived stress and distress seemed to be risk factors for the development of DM2 among men but not women. The studies on WS showed conflicting results. Overall WS seemed not to be supported as a risk factor for DM2 among either gender, nevertheless two studies supported, respectively, high demands/low decision latitude and job strain as risk factors among women.
Stress and hypertension - Fifteen studies on chronic stress and hypertension have been included. Two studies evaluated the exposure to marital stress on two different cohorts. On individual cohort one study evaluated perceived stress in general and one evaluated distress while 12 studies evaluated exposure to WS based on ten different cohorts. One study evaluated both marital stress and job strain in the same cohort (52).

Six studies evaluated BP by use of ambulatory monitoring (i.e. continuous BP monitoring), seven studies used office BP and two studies measured incident hypertension defined by start of using antihypertensive medication during follow-up. Ambulatory BP has been found to better predict clinical cardiovascular outcomes than office $\mathrm{BP}$ and is regarded as the most reliable and reproducible method (53).

Marital stress One study found that among 103 men and women, low marital cohesion and/or satisfaction was associated with a significantly but small increase in 3-year 24-h diastolic and systolic ambulatory BP (DBP and SBP) (54). The other study evaluated the effect of double exposure of both marital cohesion and job strain (52). The study found that subjects with both high job strain and low cohesive marriage had a small but significant increase in ambulatory SBP during one year, while those with high job strain and a highly cohesive marriage had a small but significant reduction in SBP during 1 year (52). However, in subjects without job strain, BP decreased during the year both among the subjects with and without cohesive marriages (52).

Perceived stress A study on 7066 men and women found that participants rating the highest level of perceived stress at baseline had a small but significantly increased risk of starting on anti-hypertensive treatment during the 10 years of follow-up (37).

Distress A study on 466 men and women did not find an association between distress and incident hypertension defined as an office BP $\geq 130 / 85$ after 6 years of follow-up (16).

Work stress Seven studies evaluated job strain of which five did not divide their results by gender: one study included 11777 men and 49145 women and found no significant association between job strain and chronic hypertension, after 3.5 years of follow-up, when hypertension was based on health insurance examinations (office BP $\geq 190 / 95$ ) (55). This null association was also found in a study on 209 men and women followed for 5 years, evaluating job

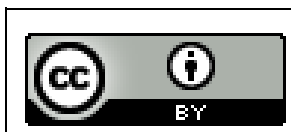

This work is licensed under a Creative Commons Attribution 3.0 Unported License. 
strain as a risk factor for increased ambulatory SBP or DBP (56). Likewise, a study based on the Whitehall II cohort found no association between job strain group and change in ambulatory SBP or DBP, among 5630 men and 2456 women followed for 11 years (57). The study on double exposure from marital cohesion and job strain, however, found a significant association between job strain and change in 24-h ambulatory BP after 1 year of follow-up (52).

Two studies on job strain divided their results by gender and both found job strain to be associated with increased office BP among men but not among women $(58,59)$. In line with these results, a study including only men found those participants that experienced high job strain at baseline but had no job strain at follow-up to have a significant decrease in mean ambulatory BP at work and at home (60). However, those with no job strain at baseline but with job strain at the 3-years follow-up and those with the same job strain exposure at both time points, exhibited no significant changes in ambulatory BP (60)

Concerning other measures of WS, one study followed 3200 men and women for 8 years and found a significantly positive association between change in job demands but not decision latitude and incident hypertension (measured by office BP levels over 160/95 or start of using antihypertensive medication during follow-up) (61). Another study followed 2357 men and women for 20 years and found that worrying about keeping ones job was significantly associated to the development of hypertension (defined as start of using antihypertensive medication during follow-up) among men, but not among women (62). In another study, a low workplace social capital was found to be associated with chronic hypertension, based on health insurance examination, among 11777 men but not among 49145 women in a study with 3.5 years of follow-up (55). The same gender difference was found when evaluating low feeling of justice at work and incident hypertension measured by office BP or start on antihypertensive treatment among 4398 men and 1923 women followed for 18 years (18). The opposite gender difference was nevertheless found regarding low job satisfaction in a cohort of 2511 men and 443 women (33). Thus, the dissatisfied women were more in risk of increased office DBP after 4-7 years, whereas no influence of job satisfaction was found on office DBP among the included men (33). Also effort-reward imbalance has been found associated with increased risk of ambulatory BP $\geq 135 / 85$ among women over 45 years old, while no association was found among younger women or among men of any age (63).
Conclusion Marital stress and perceived stress might have an influence on BP, while an association with distress was not supported. Evaluating job strain, most studies not separating their results by gender did not support an association between job strain and increase in BP, whereas the studies taking gender into account supported job strain as a risk factor for increased BP among men but not among women. Measuring other areas of WS, most studies divided the results by gender and found that worrying about keeping ones job, low workplace social capital, and low feeling of justice seemed associated with increased BP among men, while dissatisfaction with one's job and effort-reward imbalance seemed more associated with increased BP among women. This indicates that different types of WS affect the BP differently between genders. An obstacle when comparing the studies evaluating BP was the differences in methods used for evaluating BP, e.g. office or ambulatory BP or start of using antihypertensive treatment. The ways used for evaluation are highlighted in Table 5.

\section{Discussion}

Based on this literature survey, and with the reported inclusion and exclusion criteria for evaluation, we find that the majority of the studies included support an association between chronic psychosocial stress and the development of MES. With respect to weight gain stress from relationships, generally perceived stress and distress are overall supported as risk factors while the studies on WS showed conflicting results. Too few studies evaluating the development of dyslipidemia were available to draw a conclusion; however, a trend toward a positive association was present. With respect to DM2, the prospective studies supported perceived stress and distress as risk factors among men, but not among women. Regarding WS and DM2, the results were ambiguous; however, there was a tendency of no association. With respect to hypertension, perceived stress but not distress seemed associated with increased BP. Regarding WS and development of hypertension, most studies found some association, mainly among men. Intriguingly the association between the different measures of WS (e.g. job strain and effort-reward imbalance) and development of hypertension differed between men and women.

To our knowledge, this is the first systematic review evaluating chronic stress as a risk factor for MES, which precluded comparisons with previous systematic reviews.

On the individual elements of MES, previous reviews and meta-analyses have been published: a meta-analysis of longitudinal studies on stress as a risk factor for adiposity

This work is licensed under a Creative Commons Attribution 3.0 Unported License. 
found overall stress to be associated with increasing adiposity $(r=0.014, P<0.05)$ (64). However, when dividing the studies into groups of general stress and WS (job strain), neither stress groups alone showed significant associations with increasing adiposity. The meta-analyse focused more on exposure to external stressors rather than on the emotional state of stress and therefore included and excluded differently in relation to this review. Another review pooled data from 13 prospective cohort studies on the association between WS and weight gain (65). In line with the results of the Whitehall II study, they found a bidirectional association between job strain and obesity in the cross-sectional data; however, this was not tested in the longitudinal data $(32,65)$. In the longitudinal data, the study found that neither job strain at baseline nor continued job strain throughout follow-up was associated with obesity at follow-up, while development of job strain during follow-up was associated with becoming obese at follow-up (65). The results of these analyses have, however, been disputed due to methodological issues (66).

Regarding DM2 in line with this review, a review from the European Depression in Diabetes Research Consortium from 2010 found prospective epidemiological studies to support an association with general emotional stress while conflicting results were found regarding WS (67). Concerning DM2 and WS, a meta-analysis from 2012, including both cross-sectional and prospective observational epidemiological studies, supported the finding of a trend toward no association (68).

On the association between stress and the development of elevated BP, a systematic review of observational studies from 2009 included ten cohort studies and four case-control studies and found a trend for a positive association between chronic stress and elevated BP (69). Regarding job strain, another review found some association between job strain or job strain change and ambulatory BP change when including three longitudinal studies (70). Another recent review on psychosocial work factors and BP has included 40 cross-sectional, 12 prospective studies, and two case-control studies and found that approximately half of the studies supported a significant adverse effect of psychosocial work factors on $\mathrm{BP}(71)$. A more consistent adverse effect was observed among men as compared with women (71).

Theoretically, it is plausible that psychological stress induces MES. The pathway is probably multifactorial but could be explained either through stress-induced changes in the HPA axis or/and through stress-induced change in behavior. Considering hormonal changes a stress-induced hyperactivity of the HPA axis may explain the association between stress and all elements of MES: first, excess amounts of glucocorticoids enhance hepatic gluconeogenesis and inhibit the secretion and action of insulin leading to insulin resistance $(72,73)$. Second, glucocorticoids cause differentiation and proliferation of adipocytes and redistribution of fat, resulting in central adiposity, as it is seen in patients with Cushing's syndrome (74). Third, glucocorticoids decrease lipoprotein-lipase activity which lowers the level of HDL cholesterol and therefore skews the lipid-axes (75). Fourth, elevated levels of glucocorticoids are widely recognized to induce hypertension (76).

A change in behavior, due to chronic stress, may also be the pathway to MES. Kouvonen et al. (77) have found a dose dependency between WS (effort-reward imbalance) and the number of health risk behaviors (smoking, BMI $>25$, physically inactivity, and heavy drinking). People with perceived stress have been shown to spend less time on physical activity and change diet toward eating products richer in fat and sugar (78). Furthermore, stress reduces the probability of success with quitting smoking or reduce alcohol intake (37).

Human characteristics and coping mechanisms differ between individuals, causing them to react differently to stressors. The stress response therefore varies greatly among individuals and both acute and chronic stressors might be modulated with respect to personal factors. The impact of diversity in human characteristics should therefore not be underestimated when interpreting the results of this review. Several studies included finds differences between genders. This gender difference could be explained by women tending to choose other coping mechanisms than men when feeling stressed (79). Also women more often than men generally appraise everyday situations as stressful (79). Considering WS, an explanation for the gender difference could be that men and women have different occupational trajectories and have different effects of social support at work (71).

Overall, this review support that psychological stress is a causal factor for developing MES, while stress as a causal factor for the individual elements of MES shows more ambiguous results. This could indicate that MES as a defined syndrome combines the individual elements to a higher degree than just the sum of the individual elements. This argues for keeping MES as a clinical relevant phenotype even though the risk due to MES is no greater than the sum of the individual risk factors of the syndrome (80).

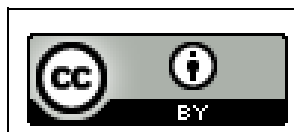

This work is licensed under a Creative Commons Attribution 3.0 Unported License. 


\section{Strengths and limitations of the studies included}

Most of the studies included had the qualities of following a large cohort over a long time. However, the studies included in this review had a varying quality. Most studies measured the stress exposure at baseline and the MES outcome at the end of follow-up though both the level of stress as well as the MES variables may have fluctuated during this period. Likewise, most studies adjusted for baseline status on physical activity, smoking, and alcohol consumption. However, this may also have changed over time as a response to changes in stress level. Overall, the included studies had a disparity in the adjustments for variables acting as covariates, confounders, or mediators, which could influence the results. Most studies included marital status, socioeconomic, and behavioral factors as physical activity. However, only one study included hours of sleep (38), though stress has been shown to be a strong predictor of sleep duration, and both short and long sleep durations are associated with elevated BMI (81). Likewise, only few studies adjusted for other exposures of stress when measuring WS and vice versa even though adjustment for the stress caused by stressors outside of the area in focus may have given a more adequate picture of the impact of the stress studied. To give an overview of the quality of the studies on this point, the variables included in the adjusted analyses are included in the Tables 1, 2, 3, 4 and 5 .

\section{Strengths and limitations of this review}

The strengths of this review are the overview of studies covering the area of chronic stress and both the MES as a syndrome as well as the individual components of the MES. To our knowledge this is the first review to give an overview of both MES as a syndrome and the elements of MES. The prospective design of the studies included provides the best basis for assessing causal relationships. Furthermore, the inclusion of studies measuring both stress in general terms, WS, and distress is strength of this review. We think, however, that it is an advantage that we in the results sections have separated the items to give a more nuanced picture of the area.

A limit specifically to this review is that the majority of studies were excluded since they were not prospective of nature, leaving only a small number of studies available for inclusion especially for dyslipidemia. Thus, one can argue that this form of systematic review is not generalizable, or that the literature is not ready for such a review. However, we believe that the 39 studies included represent sufficiently large populations for conclusions to be valid.
Also many details harbored in the studies included are omitted. This was chosen for the purpose of magnitude of this review, and we believe enough details are included to cover the essential information.

The heterogeneity of the included studies concerning both exposure, outcome, control for confounding factors, and gender distribution presents a major limitation and is the reason why this is a narrative rather than a quantitatively systematic review. Also based on the variety in control for confounding factors, we decided to include the 'must adjusted' results, which may also represent a limit to the study.

The role of psychosocial stress as a risk factor for MES and the different elements of MES are still unsettled since overall the present evidence for an association is limited. In this context, it is essential to realise that measuring stress at baseline and reporting changes over many years is a fundamental problem, since the burden might fluctuate over time. However, since a consensus on MES being a risk factor for the development of ischemic heart disease, the current evidence level seems to be sufficient to recommend an increased focus on stress-reducing acts both in workplaces and in private life. Prospective studies with specific interventions in order to reduce chronic stress are warranted as is studies focusing on the mechanisms linking chronic psychosocial stress and incident MES. In future studies, a single measure of stress, if ever possible, and a single measure of MES should be preferred.

\section{Declaration of interest}

The authors declare that there is no conflict of interest that could be perceived as prejudicing the impartiality of the review.

\section{Funding}

This review did not receive any specific grant from any funding agency in the public, commercial or not-for-profit sector.

\section{References}

1 Mottillo S, Filion KB, Genest J, Joseph L, Pilote L, Poirier P, Rinfret S, Schiffrin EL \& Eisenberg MJ. The metabolic syndrome and cardiovascular risk a systematic review and meta-analysis. Journal of the American College of Cardiology 201056 1113-1132. (doi:10.1016/j.jacc.2010.05.034)

2 Bech P. In Clinical Psychometrics. 1st Edn, 2012. Oxford, UK: Wiley Blackwell.

3 Holmes ME, Ekkekakis P \& Eisenmann JC. The physical activity, stress and metabolic syndrome triangle: a guide to unfamiliar territory for the obesity researcher. Obesity Reviews 201011 492-507. (doi:10.1111/j. 1467-789X.2009.00680.x)

4 Goldstein DS \& McEwen B. Allostasis, homeostats, and the nature of stress. Stress 20025 55-58. (doi:10.1080/102538902900012345)

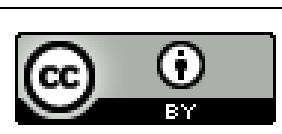

This work is licensed under a Creative Commons Attribution 3.0 Unported License. 
5 van-Holland BJ, Frings-Dresen MH \& Sluiter JK. Measuring short-term and long-term physiological stress effects by cortisol reactivity in saliva and hair. International Archives of Occupational and Environmental Health 201285 849-852. (doi:10.1007/s00420-011-0727-3)

6 Schubert C, Lambertz M, Nelesen RA, Bardwell W, Choi JB \& Dimsdale JE. Effects of stress on heart rate complexity - a comparison between short-term and chronic stress. Biological Psychology 200980 325-332. (doi:10.1016/j.biopsycho.2008.11.005)

7 Raikkonen K, Matthews KA \& Kuller LH. Depressive symptoms and stressful life events predict metabolic syndrome among middle-aged women: a comparison of World Health Organization, Adult Treatment Panel III, and International Diabetes Foundation definitions. Diabetes Care 200730 872-877. (doi:10.2337/dc06-1857)

8 Cohen S, Kamarck T \& Mermelstein R. A global measure of perceived stress. Journal of Health and Social Behavior 198324 385-396. (doi:10.2307/2136404)

9 Karasek R, Brisson C, Kawakami N, Houtman I, Bongers P \& Amick B. The Job Content Questionnaire (JCQ): an instrument for internationally comparative assessments of psychosocial job characteristics. Journal of Occupational Health Psychology 19983 322-355. (doi:10.1037/ 1076-8998.3.4.322)

10 Siegrist J, Starke D, Chandola T, Godin I, Marmot M, Niedhammer I \& Peter R. The measurement of effort-reward imbalance at work: European comparisons. Social Science \& Medicine 200458 1483-1499. (doi:10.1016/S0277-9536(03)00351-4)

11 Landsbergis P, Theorell T, Schwartz J, Greiner BA \& Krause N. Measurement of psychosocial workplace exposure variables. Occupational Medicine 200015 163-188.

12 Goldberg D \& Williams P. In A users's guide to the General Health Questionnaire. Eds DP Goldberg \& PDPM Williams. 1988. Windsor, Berks, UK: NFER-Nelson.

13 Liberati A, Altman DG, Tetzlaff J, Mulrow C, Gotzsche PC, Ioannidis JPA, Clarke M, Devereaux PJ, Kleijnen J \& Moher D. The PRISMA statement for reporting systematic reviews and meta-analyses of studies that evaluate healthcare interventions: explanation and elaboration. BMJ 2009339 b2700. (doi:10.1136/bmj.b2700)

14 Troxel WM, Matthews KA, Gallo LC \& Kuller LH. Marital quality and occurrence of the metabolic syndrome in women. Archives of Internal Medicine 2005165 1022-1027. (doi:10.1001/archinte.165.9.1022)

15 Whisman MA \& Uebelacker LA. A longitudinal investigation of marital adjustment as a risk factor for metabolic syndrome. Health Psychology 201231 80-86. (doi:10.1037/a0025671)

16 Puustinen PJ, Koponen H, Kautiainen H, Mantyselka P \& Vanhala M. Psychological distress predicts the development of the metabolic syndrome: a prospective population-based study. Psychosomatic Medicine 201173 158-165. (doi:10.1097/PSY.0b013e3182037315)

17 Chandola T, Brunner E \& Marmot M. Chronic stress at work and the metabolic syndrome: prospective study. BMJ 2006332 521-525. (doi:10.1136/bmj.38693.435301.80)

18 Gimeno D, Tabák AG, Ferrie JE, Shipley MJ, De Vogli R, Elovainio M M, Vahtera J, Marmot MG \& Kivimäki M. Justice at work and metabolic syndrome: the Whitehall II study. Occupational and Environmental Medicine 201067 256-262. (doi:10.1136/oem .2009.047324)

19 Edwards EM, Stuver SO, Heeren TC \& Fredman L. Job strain and incident metabolic syndrome over 5 years of follow-up: the coronary artery risk development in young adults study. Journal of Occupational and Environmental Medicine 201254 1447-1452. (doi:10.1097/JOM. Ob013e3182783f27)

20 Kouvonen A, Stafford M, De Vogli R, Shipley MJ, Marmot MG, Cox T, Vahtera J, Väänänen A, Heponiemi T, Singh-Manoux A et al. Negative aspects of close relationships as a predictor of increased body mass index and waist circumference: the Whitehall II study. American Journal of Public Health 2011101 1474-1480. (doi:10.2105/AJPH.2010.300115)

21 Block JP, He Y, Zaslavsky AM, Ding L \& Ayanian JZ. Psychosocial stress and change in weight among US adults. American Journal of Epidemiology 2009170 181-192. (doi:10.1093/aje/kwp104)
22 Fowler-Brown AG, Bennett GG, Goodman MS, Wee CC, CorbieSmith GM \& James SA. Psychosocial stress and 13-year BMI change among blacks: the Pitt County Study. Obesity 200917 2106-2109. (doi:10.1038/oby.2009.130)

23 Gerace TA \& George VA. Predictors of weight increases over 7 years in fire fighters and paramedics. Preventive Medicine 199625 593-600. (doi:10.1006/pmed.1996.0094)

24 Korkeila M, Kaprio J, Rissanen A, Koshenvuo M \& Sorensen TI. Predictors of major weight gain in adult Finns: stress, life satisfaction and personality traits. International Journal of Obesity and Related Metabolic Disorders 199822 949-957. (doi:10.1038/sj.ijo.0800694)

25 Magee CA, Caputi P, Stefanic N \& Iverson DC. Occupational factors associated with 4-year weight gain in Australian adults. Journal of Occupational and Environmental Medicine 201052 977-981. (doi:10.1097/JOM.0b013e3181f26e72)

26 Allard KO, Thomsen JF, Mikkelsen S, Rugulies R, Mors O, Kaergaard A, Kolstad HA, Kaerlev L, Andersen JH, Hansen AM et al. Effects of psychosocial work factors on lifestyle changes: a cohort study. Journal of Occupational and Environmental Medicine 201153 1364-1371. (doi:10.1097/JOM.0b013e3182363bda)

27 Berset M, Semmer NK, Elfering A, Jacobshagen N \& Meier LL. Does stress at work make you gain weight? A two-year longitudinal study Scandinavian Journal of Work, Environment \& Health 201137 45-53. (doi:10.5271/sjweh.3089)

28 Kivimaki M, Leino-Arjas P, Luukkonen R, Riihimaki H, Vahtera J \& Kirjonen J. Work stress and risk of cardiovascular mortality: prospective cohort study of industrial employees. BMJ 2002325 857. (doi:10.1136/ bmj.325.7369.857)

29 Roos E, Lallukka T, Rahkonen O, Lahelma E \& Laaksonen M. Working conditions and major weight gain-a prospective cohort study. Archives of Environmental \& Occupational Health 201368 166-172. (doi:10.1080/ 19338244.2012.686931)

30 Lallukka T, Sarlio-Lahteenkorva S, Kaila-Kangas L, Pitkaniemi J, Luukkonen R \& Leino-Arjas P. Working conditions and weight gain: a 28-year follow-up study of industrial employees. European Journal of Epidemiology 200823 303-310. (doi:10.1007/s10654-008-9233-7)

31 Ishizaki M, Nakagawa H, Morikawa Y, Honda R, Yamada Y \& Kawakami N. Influence of job strain on changes in body mass index and waist circumference-6-year longitudinal study. Scandinavian Journal of Work, Environment \& Health 200834 288-296. (doi:10.5271/ sjweh.1267)

32 Kivimaki M, Head J, Ferrie JE, Shipley MJ, Brunner E, Vahtera J \& Marmot MG. Work stress, weight gain and weight loss: evidence for bidirectional effects of job strain on body mass index in the Whitehall II study. International Journal of Obesity 200630 982-987. (doi:10.1038/sj. ijo.0803229)

33 Heslop P, Smith GD, Metcalfe C, Macleod J \& Hart C. Change in job satisfaction, and its association with self-reported stress, cardiovascular risk factors and mortality. Social Science \& Medicine 200254 1589-1599. (doi:10.1016/S0277-9536(01)00138-1)

34 Brunner EJ, Chandola T \& Marmot MG. Prospective effect of job strain on general and central obesity in the Whitehall II study. American Journal of Epidemiology 2007165 828-837. (doi:10.1093/aje/kwk058)

35 Gram QH, Christensen U, Christensen KB, Aust B, Borg V \& Bjorner JB. Psychosocial work environment factors and weight change: a prospective study among Danish health care workers. BMC Public Health 201313 43. (doi:10.1186/1471-2458-13-43)

36 Eek F \& Ostergren PO. Factors associated with BMI change over five years in a Swedish adult population. Results from the Scania Public Health Cohort Study. Scandinavian Journal of Public Health 200937 532-544. (doi:10.1177/1403494809104359)

37 Rod NH, Gronbaek M, Schnohr P, Prescott E \& Kristensen TS. Perceived stress as a risk factor for changes in health behaviour and cardiac risk profile: a longitudinal study. Journal of Internal Medicine 2009266 467-475. (doi:10.1111/j.1365-2796.2009.02124.x)

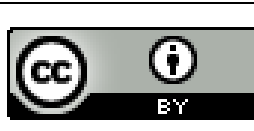


38 Kato M, Noda M, Inoue M, Kadowaki T \& Tsugane S. Psychological factors, coffee and risk of diabetes mellitus among middle-aged Japanese: a population-based prospective study in the JPHC study cohort. Endocrine Journal 200956 459-468. (doi:10.1507/endocrj.K09E-003)

39 Toshihiro M, Saito K, Takikawa S, Takebe N, Onoda T \& Satoh J. Psychosocial factors are independent risk factors for the development of type 2 diabetes in Japanese workers with impaired fasting glucose and/or impaired glucose tolerance,. Diabetic Medicine 200825 1211-1217. (doi:10.1111/j.1464-5491.2008.02566.x)

40 Novak M, Bjorck L, Giang KW, Heden-Stahl C, Wilhelmsen L \& Rosengren A. Perceived stress and incidence of type 2 diabetes: a 35-year follow-up study of middle-aged Swedish men. Diabetic Medicine 201330 e8-16. (doi:10.1111/dme.12037)

41 Williams ED, Magliano DJ, Tapp RJ, Oldenburg BF \& Shaw JE. Psychosocial stress predicts abnormal glucose metabolism: the Australian Diabetes, Obesity and Lifestyle (AusDiab) study. Annals of Behavioral Medicine 201346 62-72. (doi:10.1007/s12160-013-9473-y)

42 Eriksson AK, Ekbom A, Granath F, Hilding A, Efendic S \& Ostenson CG. Psychological distress and risk of pre-diabetes and type 2 diabetes in a prospective study of Swedish middle-aged men and women. Diabetic Medicine 200825 834-842. (doi:10.1111/j.1464-5491.2008.02463.x)

43 Mommersteeg PM, Herr R, Zijlstra WP, Schneider S \& Pouwer F. Higher levels of psychological distress are associated with a higher risk of incident diabetes during 18 year follow-up: results from the British Household Panel Survey. BMC Public Health 2012121109 (doi:10.1186/1471-2458-12-1109)

44 Eriksson AK, van den Donk M, Hilding A \& Ostenson CG. Work stress, sense of coherence, and risk of type 2 diabetes in a prospective study of middle-aged Swedish men and women. Diabetes Care 201336 2683-2689. (doi:10.2337/dc12-1738)

45 Brunner EJ \& Kivimaki M. Epidemiology: work-related stress and the risk of type 2 diabetes mellitus. Nature Reviews. Endocrinology 20139 449-450. (doi:10.1038/nrendo.2013.124)

46 Heraclides AM, Chandola T, Witte DR \& Brunner EJ. Work stress, obesity and the risk of type 2 diabetes: gender-specific bidirectional effect in the Whitehall II study. Obesity 201120 (428) 33. (doi:10.1038/ oby.2011.95)

47 Kawakami N, Araki S, Takatsuka N, Shimizu H \& Ishibashi H. Overtime, psychosocial working conditions, and occurrence of non-insulin dependent diabetes mellitus in Japanese men. Journal of Epidemiology and Community Health 199953 359-363. (doi:10.1136/jech.53.6.359)

48 Kroenke CH, Spiegelman D, Manson J, Schernhammer ES, Colditz GA \& Kawachi I. Work characteristics and incidence of type 2 diabetes in women. American Journal of Epidemiology 2007165 175-183. (doi:10.1093/aje/kwj355)

49 Smith PM, Glazier RH, Lu H \& Mustard CA. The psychosocial work environment and incident diabetes in Ontario, Canada. Occupational Medicine 201262 413-419. (doi:10.1093/occmed/kqs128)

50 Kumari M, Head J \& Marmot M. Prospective study of social and other risk factors for incidence of type 2 diabetes in the Whitehall II study. Archives of Internal Medicine 2004164 1873-1880. (doi:10.1001/ archinte.164.17.1873)

51 Toker S, Shirom A, Melamed S \& Armon G. Work characteristics as predictors of diabetes incidence among apparently healthy employees. Journal of Occupational Health Psychology 201217 259-267. (doi:10.1037/a0028401)

52 Tobe SW, Kiss A, Sainsbury S, Jesin M, Geerts R \& Baker B. The impact of job strain and marital cohesion on ambulatory blood pressure during 1 year: the double exposure study. American Journal of Hypertension 2007 20 148-153. (doi:10.1016/j.amjhyper.2006.07.011)

53 Myers MG, Haynes RB \& Rabkin SW. Canadian hypertension society guidelines for ambulatory blood pressure monitoring. American Journal of Hypertension 199912 1149-1157. (doi:10.1016/S08957061(99)00199-5)

54 Baker B, Paquette M, Szalai JP, Driver H, Perger T, Helmers K, O'Kelly B $\&$ Tobe $\mathrm{S}$. The influence of marital adjustment on 3-year left ventricular mass and ambulatory blood pressure in mild hypertension. Archives of Internal Medicine 2000160 3453-3458. (doi:10.1001/ archinte.160.22.3453)

55 Oksanen T, Kawachi I, Jokela M, Kouvonen A, Suzuki E, Takao S, Virtanen M, Pentti J, Vahtera J \& Kivimäki M. Workplace social capital and risk of chronic and severe hypertension: a cohort study. Journal of Hypertension 201230 1129-1136. (doi:10.1097/HJH. Ob013e32835377ed)

56 Fauvel JP, M'Pio I, Quelin P, Rigaud JP, Laville M \& Ducher M. Neither perceived job stress nor individual cardiovascular reactivity predict high blood pressure. Hypertension 200342 1112-1116. (doi:10.1161/01. HYP.0000102862.93418.EE)

57 Kivimaki M, Head J, Ferrie JE, Shipley MJ, Steptoe A, Vahtera J \& Marmot MG. Hypertension is not the link between job strain and coronary heart disease in the Whitehall II study. American Journal of Hypertension 200720 1146-1153.

58 Guimont C, Brisson C, Dagenais GR, Milot A, Vézina M, Masse B, Moisan J, Laflamme N \& Blanchette C. Effects of job strain on blood pressure: a prospective study of male and female white-collar workers. American Journal of Public Health 200696 1436-1443. (doi:10.2105/ AJPH.2004.057679)

59 Ohlin B, Berglund G, Rosvall M \& Nilsson PM. Job strain in men, but not in women, predicts a significant rise in blood pressure after 6.5 years of follow-up. Journal of Hypertension 200725 525-531. (doi:10.1097/HJH.0b013e32801220fa)

60 Schnall PL, Schwartz JE, Landsbergis PA, Warren K \& Pickering TG. A longitudinal study of job strain and ambulatory blood pressure: results from a three-year follow-up. Psychosomatic Medicine $1998 \mathbf{6 0}$ 697-706.

61 Markovitz JH, Matthews KA, Whooley M, Lewis CE \& Greenlund KJ. Increases in job strain are associated with incident hypertension in the CARDIA Study. Annals of Behavioral Medicine 200428 4-9. (doi:10.1207/ s15324796abm2801_2)

62 Levenstein S, Smith MW \& Kaplan GA. Psychosocial predictors of hypertension in men and women. Archives of Internal Medicine 2001161 1341-1346. (doi:10.1001/archinte.161.10.1341)

63 Gilbert-Ouimet M, Brisson C, Vezina M, Milot A \& Blanchette C. Repeated exposure to effort-reward imbalance, increased blood pressure, and hypertension incidence among white-collar workers: effort-reward imbalance and blood pressure. Journal of Psychosomatic Research 201272 26-32. (doi:10.1016/j.jpsychores.2011.07.002)

64 Wardle J, Chida Y, Gibson EL, Whitaker KL \& Steptoe A. Stress and adiposity: a meta-analysis of longitudinal studies. Obesity 201119 771-778. (doi:10.1038/oby.2010.241)

65 Nyberg ST, Heikkila K, Fransson EI, Alfredsson L, De Bacquer D, Bjorner JB, Bonenfant S, Borritz M, Burr H, Casini A et al. Job strain in relation to body mass index: pooled analysis of 160000 adults from 13 cohort studies. Journal of Internal Medicine 2012272 65-73. (doi:10.1111/j.1365-2796.2011.02482.x)

66 Choi B, Dobson M, Landsbergis P, Ko S, Yang H, Schnall P \& Baker D. Job strain and obesity. Journal of Internal Medicine 2014275 438-440. (doi:10.1111/joim.12173)

67 Pouwer F, Kupper N \& Adriaanse MC. Does emotional stress cause type 2 diabetes mellitus? A review from the European Depression in Diabetes (EDID) Research Consortium Discovery Medicine 20109 112-118.

68 Cosgrove MP, Sargeant LA, Caleyachetty R \& Griffin SJ. Work-related stress and type 2 diabetes: systematic review and meta-analysis. Occupational Medicine 201262 167-73. (doi:10.1093/occmed/kqs002)

69 Sparrenberger F, Cichelero FT, Ascoli AM, Fonseca FP, Weiss G, Berwanger O, Fuchs SC, Moreira LB \& Fuchs FD. Does psychosocial stress cause hypertension? A systematic review of observational studies Journal of Human Hypertension 200923 12-19. (doi:10.1038/jhh.2008.74)

70 Landsbergis PA, Dobson M, Koutsouras G \& Schnall P. Job strain and ambulatory blood pressure: a meta-analysis and systematic review. American Journal of Public Health 2013103 e61-e71. (doi:10.2105/AJPH. 2012.301153)

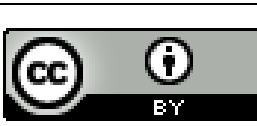

This work is licensed under a Creative Commons Attribution 3.0 Unported License. 
71 Gilbert-Ouimet M, Trudel X, Brisson C, Milot A \& Vezina M. Adverse effects of psychosocial work factors on blood pressure: systematic review of studies on demand-control-support and effort-reward imbalance models. Scandinavian Journal of Work, Environment \& Health 201440 109-132. (doi:10.5271/sjweh.3390)

72 Bjorntorp P. Neuroendocrine perturbations as a cause of insulin resistance. Diabetes/Metabolism Research and Reviews 199915 427-441. (doi:10.1002/(SICI)1520-7560(199911/12)15:6 < 427::AID-DMRR68> 3.0.CO;2-C)

73 Rosmond R. Role of stress in the pathogenesis of the metabolic syndrome. Psychoneuroendocrinology 200530 1-10.

74 Macfarlane DP, Forbes S \& Walker BR. Glucocorticoids and fatty acid metabolism in humans: fuelling fat redistribution in the metabolic syndrome. Journal of Endocrinology 2008197 189-204. (doi:10.1677/ JOE-08-0054)

75 Niaura R, Stoney CM \& Herbert PN. Lipids in psychological research: the last decade. Biological Psychology 199234 1-43. (doi:10.1016/03010511(92)90022-M)

76 Goodwin JE \& Geller DS. Glucocorticoid-induced hypertension. Pediatric Nephrology 201127 1059-66. (doi:10.1007/s00467-011-1928-4)
77 Kouvonen A, Kivimaki M, Virtanen M, Heponiemi T, Elovainio M, Pentti J, Linna A \& Vahtera J. Effort-reward imbalance at work and the co-occurrence of lifestyle risk factors: cross-sectional survey in a sample of 36,127 public sector employees. BMC Public Health 2006624. (doi:10.1186/1471-2458-6-24)

78 Zellner DA, Loaiza S, Gonzalez Z, Pita J, Morales J, Pecora D \& Wolf A. Food selection changes under stress. Physiology \& Behaviour 200687 789-793. (doi:10.1016/j.physbeh.2006.01.014)

79 Pilar Matud M. Gender differences in stress and coping styles. Personality and Individual Differences 200437 1401-1415. (doi:10.1016/j. paid.2004.01.010)

80 Mente A, Yusuf S, Islam S, McQueen MJ, Tanomsup S, Onen CL, Rangarajan S, Gerstein HC, Anand SS \& INTERHEART Investigators . Metabolic syndrome and risk of acute myocardial infarction a case-control study of 26,903 subjects from 52 countries. Journal of the American College of Cardiology 201055 2390-2398. (doi:10.1016/j.jacc. 2009.12.053)

81 Dweck JS, Jenkins SM \& Nolan LJ. The role of emotional eating and stress in the influence of short sleep on food consumption. Appetite 201372 106-113. (doi:10.1016/j.appet.2013.10.001)

Received in final form 7 April 2014

Accepted 17 April 2014 http://www.endocrineconnections.org DOI: 10.1530/EC-14-0031
(C) 2014 The authors Published by Bioscientifica Ltd

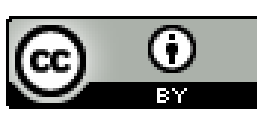

This work is licensed under a Creative Commons Attribution 3.0 Unported License. 